THSONIAN

MaPATY 


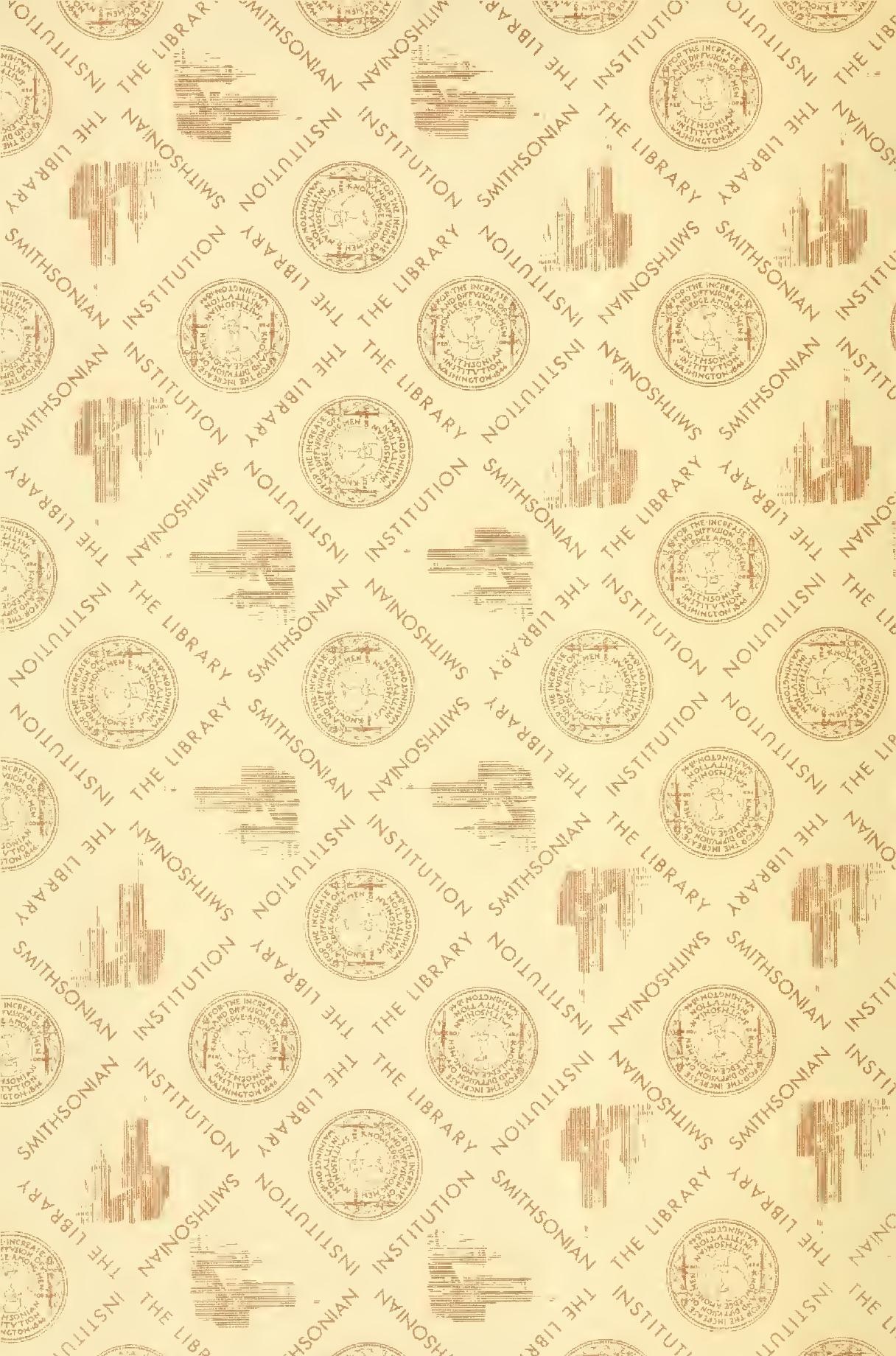







\section{SPECLAL REPORT}

OF ТНB

LNITED STATES BOARD ON GROGRAPHIC NAMUSS

RELATING TO

\section{THE GEOGRAPHIC NAMES IN THE PHILIPPINE ISLANDS.}

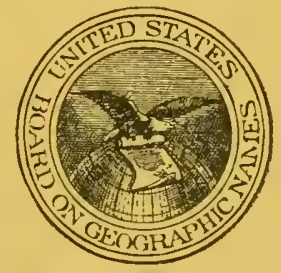

W ASHINGTON:

GOVERNMENT PRINTING OFFICE.

MAY, 1901. 

Bampliments of

Qtis O. Oasmir,

Gurator of the Qivision of Ëthrelogy

Omithonian Onstitutiase

Apreded States diationat Offuseum. 



\section{SPECIAL REPORT}

OF THE

\section{LVITED STITES BOARD ON GEOGREPHIC NAUES}

RELATING TO

\section{THE GEOGRAPHIC NAIES IN THE PHILIPPINE ISLANISS.}

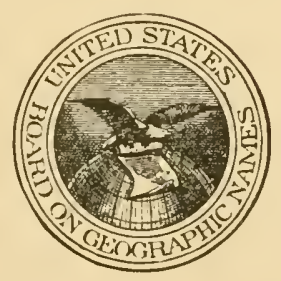

WASHINGTON:

GOVERNMENT PRINTING OFFICE.

II $;, 1901$. 
IN THE SExate of the I Xiten States,

Fobruary $28,1901$.

Resotred, That there be printed fifteen thomand copies of the special report of the United states Board on Geographic Names relating to geographic names in the Philippine Islands, transmitted to Congress ly the President February twenty-first, nineteen hundred and one; three thousand copies of which shall he for the use of the senate, five thousand copies for the use of the Honse of Representatives, and seven thousand copies for distribution by said board among the ciovernment lepartments, public libraries, and other suitable teposituries.

Attest:

Cinarles G. Bennett,

Secretur\%. 
To the Senate and House of Representutives:

I transmit herewith for the information of the Congress and with a view to its publication in suitable form. if such action is deemed desirable. a special report of the L'nited States Board on Geographic Names, relating to geographic names in the Philippine Islands, and invite attention to the recommendation of the board:

"That in aldition to the usual number there be printed 15,000 copies; 2,000 copies for the use of the Senate; 3,000 copies for the use of the House of Representatives, and 10,000 copies for distribution by the board to the Executive Departments and the public."

Executive Maxalon.

William MCKInLeY.

Febreury 21, 1901. 

LIST OF COASTIISE NANES, PHILIPPINE ARCHIPELAGO, AS CONPILED IN THE T. s. HYDROGRPHIC OFFICE. 1901. 

To the President:

I have the honor to lay before you a special report of the United States Board on Geographic Names relative to geographic names in the Philippine Iskands. It consists of a list showing the approved spelling of about 4,000 coastwise names in the Philippine Archipelago, and its printing and distribution is urgently needed by the Executive Departments.

The board respectfully recommends:

That in addition to the usual number there be printed 15,000 copies; 2,000 copies for the use of the Senate, 3,000 copies for the use of the House of Representatives, and 10,000 copies for distribution by the board to the Exeeutive Departments and to the public.

Respectfully subunitted.

Wasmongtos, D. C., Fetorerry $21,1901$.

Henry Gannett,

Chairman. 



\section{NAIES OF COASTIWISE FEATIRES IN THE PHILIPPINE ISLANIS.}

\section{INTRODUCTION.}

Upon the aequisition of the Philippine Islands by the United States in 1598, the Hydrographic Othice was promptly called upon for charts, sailing directions, ete. of these new possessions. To meet this demand it at once began their preparation. At the outset it met much difficulty with the names. Existing charts, books, maps, and publieations disagreed, both as to names and their spelling. Spanish charts contained either all Spanish names or Spanish names and also Malay names written aceording to Spanish methods. On English charts the spelling of some of the Malay names had been altered to eonform to English and Ameriean methods of writing native names. In doing this, errors and confusion had arisen.

In this eonfusion the Hrdrographic Office appealed to the Board on Geographic Names and asked for advice. The Board considered the general question at some length. and then reeommended that the mames in eurrent use and their spelling, as shown on the hest Spanish oflicial maps and charts. should be followed. The Hydrographie Oftice followed this advice, and under the direction of Capt. C. C. Todd. hydrographer, U. S. N., prepared, chiefly from Spanish ofticial charts, the following list of about 4.000 coastwise names.

Meanwhile another list of about 6,000 Philippine geographic names had been prepared in Manilá. and in Washington. D. C.. under the direction of Rev. José Algue, S. .J.. director of the Jesuit Observatory at Manila. Those names, arranged in three groups and accompanied by 30 maps, were submitted to the United States Coast and Creodetic. Survey, and have reeently been printed by that Surver as an intro-

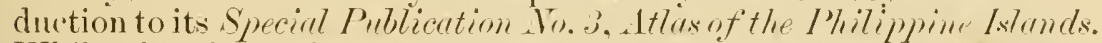
While this Coast survey publieation was going through the press, Father Algue was in Washington and read the proof. Serelal humdred of the names contained in it are also contained in this list. On the other hand, this list is much fuller for coastwise names, containing about 2,600 names not found in the Coast Survey atlas. After Father Algue had read and colreeted the proofs of the naries in the Coast Survey atlas, he kindly undertook to revise the manuscript of this list, and this to bring the two into harmony. In such revision, only 33 differences of spelling were cletected, and these for the most part trifling. In this list the spelling of those 33 names has been made to agree with the Coast Survey atlas, and aceordingly the spelling of names in that atlas and in this list are. errors exeepted, the same.

Pronuneiation is not considered in this report, which was prepared as a reference list for spelling only. Therefore all accents and diacritical marks, sinee they relate to pronunciation only, are here omitted. 
Such is not the case, however, with the Coast Survey atlas. which contains accents and is preceded by a note (p. 7) written by Father Algue and entitled The Pronmeiation of Spanish. That note is as follows:

THE PLONIXIATIUN OF SPANISH.

The Spanish language has but one acent, ealled acento agudo, which is placed over the vowels $a, e, i, o, u$; it serves to lengthen the syllable and to accentuate it more distinctly and forcibly than the other vowels.

As a general rule, all spanish words ending in a vowel are arcentuated on the penultimate, or the last syllable but one; they do not, therefore, require the accent, bat if any other syllable is to be accented it is indicated.

With the exception of proper names and adverbs, most worls encling in a consonant have the accent on the last syllable.

The Spanish a always has the sound of the English $"$ in father; the p that of ay in English; the $i$ that of ee; the $o$ that of $o h$; and the $u$ that of $o 0$, as in bort, root, etc.

The vowels in Spanish must always be fully and distinetly prononncerl, but the $u$ is silent in the svllables gue, gui, que, qui, which are pronounced gay, gee, kay, lier.

The Spanish if has a souncl nearly equivalent to the Enghish i; the z exactly that of English th, as also the Spanish " when it comes before $e$ or $i$. All the other con-onants are sounded as in English except $l l$, which is prononnced as ly in English.

In the English interpretation of the sovmil of the Spanish words the th has always the lisping somind of these letters in thin, with, ete.

The main difficulty in pronomeing spanish is the $j$ (boetah), which has the sound of $h$ very strongly aspiraterl.

The relation of the United States Board on Geographic Names to the names in the Coast survey atlas is set forth in the following extratet from a report adopted by the Board on February 2019 1901:

Hhorets the Coast and Geoletic Survey is about to issue an atlas of the l'hilippine Islands composed of maps matle at the Manila Observatory moler the direction of Rev. Father Algue, S. J.; and

Whereas this atlas contains in its introrluction three lists of geographic namen, aggregating abont 6,000 , whose spelling has been carefully revised by Father Algue to bring it into harmony with local Spanish usage in the Philippines; and

Wheres the Coast and Creorletic Survey has, by its mperintendent, asked that these names be arlopted by the Buarl as the standard for fovernment publications in so far as they affect the charts of the Philipuines; and

Wherens the Board, after careful consideration, is of opinion that these lists, together with the list prepared by the Hydrographic Office, constitute the most trustworthy information now obtainable as to the spelling of these names: Therefore

Lirsolied, That the Buard provisionally approve the spelling as given in these lists, this approval extending to spelling only and not to pronumeiation or accents by which pronunciation is indieater; it being unclerstoot that such provisional adoption shall be final, except as to such of them as shall hereatter be presenterl to the Board as disputed cases, these disputed cases to be treated as in effert wholly new. 


\section{NAMES OF' COASTWISE FEATURES IN THE PHILIPPINE ISLANDS.}

NotE.-The names are in black-faee type. These are followed by the Spanish designations of the features namer. The menning of most of them is apparent. For convenience their translation is given herewith:

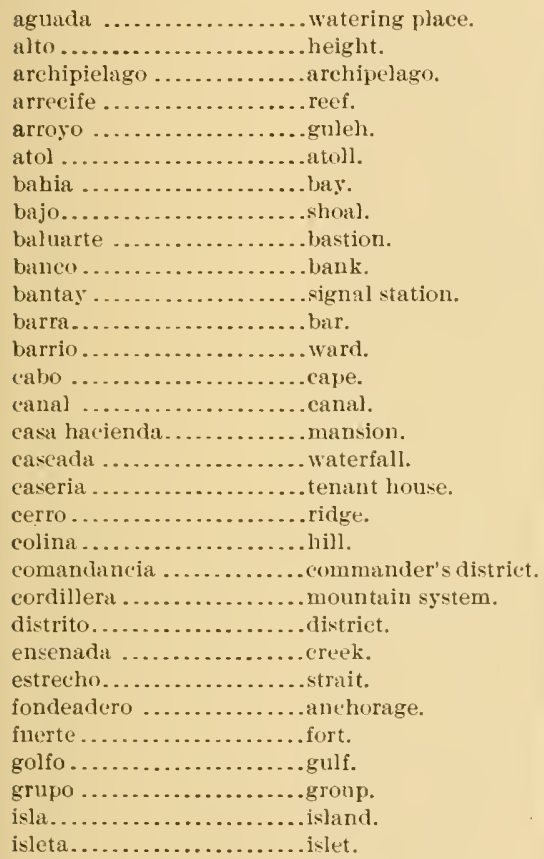

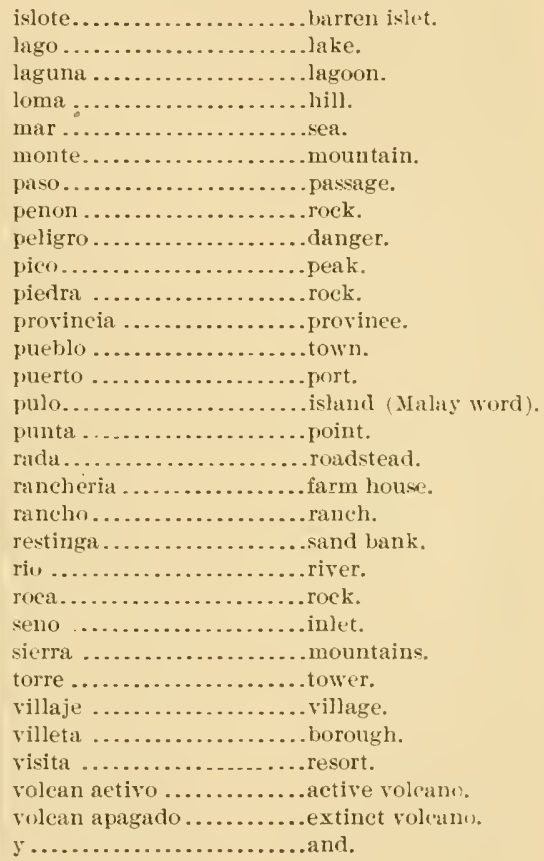

Aanosa, villaje.

Abaang, punta.

Abac, villaje.

Abagata, ensenada.

Abalili, rio.

Abalit, villaje.

Abangay, rio.

Abas, rio.

Abatan, rio.

Abdule, monte.

Abian, rio.

Abijao, rio, y villeta.

Abinolong, rio.

Abocayan, puerto.

Abongabon, punta, y rio.

Aboslan, villaje.

Abra, pueblo, y rio.

Abra de Ilog, ensenarla.

Abual, rio.

Abucay, villaje.

Abug, rio.

Abullo, villaje.

Abulug, monte, pueblo, y rio.

Abungan, rio.

Abutaon, rio.

Abuygo, rio.

Acagasan, punta.

Acal, punta.

Acantilada, punta.

Acantilado, cabo.

Acha, bajo, y punta.

Aclan, barra, punta, y rio. 
Acta, punta.

Ada, arreeife.

Adcalayo, islote.

Adela, bajo.

Adyagan, atol.

Afuera, hanco.

Afuon or Aliran or Abuluan, rio.

Againg, punta.

Aganmala, monte.

Agantao, punta.

Agauan, punta, y rio.

Agayub, rio.

Agbatan, punta, y rio.

Agbuay, punta.

Agcauayan, ensenada.

Agio, punta.

Agla, punta.

Aglacaigan, rio.

Agnacon, rio.

Agnas, punta.

Agno, rio.

Agno Chico, rio.

Agno Grande, punta, y rio.

Agoho, punta.

Agojo, punta.

Agonoy, isla, y villaje.

Agroo, pueblo.

Agros, rio.

Agosoen, punta.

Agpitan, isla.

Agram, rio.

Agria, punta.

Agsabo, rio.

Agta, punta.

Aguada, ensenada.

Aguada, (le la) punta.

Aguado, monte.

Aguaguan, punta.

Aguasa, ensenada.

Agudo, monte.

Aguirre, lanco.

Agus, rio.

Agusan, rio, y villaje.

Agutay, punta.

Agutaya, isleta, y pueblo.

Agutayan, isla.

Ajus, punta.

Ajuy, pueblo.

Alabat, isla, y punta.

Alad, isla.

Alag, puerto.

Alambaybay, seno.

Alasa, rio.

Alavigan, punta.
Albaguen, isleta.

Albay, seno, y volcan.

Albion, cabo, ensenada, y punta.

Albuera, pueblo.

Alburquerque, pueblo.

Alcala, pueblo.

Alcantara, pueblo.

Alcoy, pueblo.

Aldea, bahia, piedra, y villaje.

Alegria, pueblo, y rio.

Alerta, isla.

Aletas de Tiburon, bahia.

Aleu-leu, monte.

Alibatan, isleta.

Alibijaban, isla.

Alican, punta.

Aligbay, isla.

Alihanon, rio.

Alijara, isla.

Alimanga, punta.

Alimango, ensenada.

Alimpago, punta.

Alimpapayo, punta.

Alimpaya, punta.

Alimudin, punta.

Alincacadao, isla.

Alingsulung, punta.

Alinin, punta.

Alinit, rio.

Alipayao, villaje.

Alisut, punta.

Alitagtag, punta.

Almeria, pueblo.

Alo, punta.

Aloguinsan, puehlo.

Alon-on, punta.

Aloran, pneblo.

Alpaco, monte, y rio.

Alto, pico.

Alto del Caraballo, monte.

Alu, punta.

Alubijit, fondeadero.

Alulayan, punta.

Alungugan, punta.

Alutaya, bajo.

Aluyan, isla.

Aluyog, villaje.

Amagadpayat, islote.

Amangbucale, punta.

Amangtarang, punta.

Amanpapay, punta.

Amayat, villeta.

Amayon, monte.

Ambacon, bantay. 
Amban, punta.

Ambil, lanco, fondealero, gruporle islas, isla, y jaso.

Amblau, punta, y villeta.

Ambolon, "amai, grupo de islas, y islat.

Ambubuyo, monte.

Ambugan, isla.

Amburayan, rio.

Amo, rie.

Amogotacia, punta.

Amogotado, monte.

Amol, puntil.

Among, rio.

Amoraon, puntal.

Amoron, punta.

Amoroy, riı.

Amulon, punta.

Amulung, puehlo.

Amusic, rio.

Ana, rio, y seno.

Anaganahao, isleta, y punta.

Anahao, islute.

Anaja, rio.

Anajao, punti.

Anajauan, isla.

Anajulan, rancho.

Anambogon, punta.

Anato, isleta, y pieo.

Anauayan, grupo de islas, y isla.

Anayan, rio.

Anda, puejlo.

Andaganan, rio.

Andagao, pueblo.

Andang, monte.

Andeyro, punta.

Andis, or Ando, isla.

Andrao, isla.

Anepahan, picos.

Angalacan, rio.

Angas, punta.

Angat, rio.

Angil, punta.

Aniauan, rio.

Anibavan, rio.

Anibun, punta.

Anibungan, rio.

Anilao, pueblo, y rio.

Anilon, isla.

Aniningan, punta.

Aniniy, puelslo, y punta.

Anislagon, rio.

Anitaguipan, punta.

Antabaan, punta, rio, y seno.

Antagtacan, punta.
Antal, sent.

Antipolo, puedslo, y punt:.

Antique, jueblo, y rio.

Antiyao, rio.

Antonia, isla.

Antucao, pminta.

Apalan, rio.

Apalant, junta.

Apalasit, 1unt:ı.

Apanican, rio.

Aparri, puerto, punta, y rio.

Apat, ensenadis.

Apga, punta.

Apiat, punta, y rio.

Apit, grupo ile islas.

Apo, bajo, isla, monte', y paso.

Apud, villeta.

Apunan, punta.

Apurauan, Jalıia, fomleader'r, y punta.

Aquitabangal, rio.

Arabinag, rio.

Arado, punta.

Arambi, rio.

Arana, arrecife.

Arasasan, rio.

Arauis, punta.

Arayat, pueblo, y volean apalgarlo.

Arbol, pico.

Arbol Balete, villaje.

Arboledan, punta.

Arbusto, punta.

Aregita, islat.

Arena, isla, y punta.

Arenas, punta.

Arevalo, puehlo, y +'nu.

Arganan, rio.

Argao, pueblo, y punta.

Argos, bajo, y punta.

Arguelles, puelolo.

Arida, isla.

Aringay, pueblo.

Arnay, rio.

Aroroy, villeta.

Arouan, punta, y rio.

Asaan, punta.

Asgad, punta.

Asid, rio, y seno.

Aslom, isla.

Aslum, lago.

Asluman, punta, y rio.

Aspera, junta.

Asturias, pueblo.

Asuang, punta.

Asuero, punta. 
Asug, punta.

Ataa, isla.

Atalayan, isla.

Ateca, punta.

Atimonan, puehlo, y punta.

Aton-aton, rio.

Aubarede, bajo, y punta.

Auqui, isla.

Ausan, punta.

Ayala, pueblo.

Ayat, seno.

Ayninan, isla.

Ayoni, seno.

Ayungon, pueblo.

Azagra, villaje.

Azufre, punta.

Baao, lago, y pueblo.

Baaquec, junta, y seno.

Baay, rí.

Babangun, punta.

Babatgun, punta, y seno.

Babdalao, ensenar?a.

Babugon, isla.

Babulgan, ensenada, y punta.

Babuyan, isla, y volean activo.

Babuyanes, grupo de islas.

Bacabac, isla.

Bacacay, isla, y (de) sierra.

Bacahuan, villaje.

Bacal, punta.

Bacalan, pueblo, y rio.

Bacalaya, rio.

Bacao, isla.

Bacarra, pueblo, y puerto.

Bacat, fuerte, y rio.

Bacauan, rio.

Bacayacan, ensenala.

Bacayauan, monte.

Bac-hao, rio.

Bacian, villaje.

Bacjao, punta.

Baclayon, putblo.

Bacluc, punta.

Baclut, punta.

Baco, rio.

Bacod, villaje.

Bacol, isla.

Bacolod, capital.

Bacon, pueblo, y rio.

Bacong, junta.

Bacoo, pueblo.

Bacood, punta, y seno.

Bacoor, ensenadi, lueblo, y rio.

Bacos, isletas.
Bacot, rio.

Bacquil, rio.

Bacsal, isla.

Bacud, puntar.

Bacuit, hahia, y pueblo.

Baculin, punta, y villeta.

Bacun, estero.

Bacungan, isla.

Bacuyangan, punta.

Badajoz, meblo.

Badas, villije.

Badbalao, ensenada.

Badbaran, rio.

Badia, isla.

Badian, ensenada, fondeadero, isla, pueblo, punta, y rio.

Badoc, isla, y villaje.

Badol, villaje.

Badungbadung, islote.

Baeto, puntis.

Bagababoy, islas.

Bagabu, isla.

Bagac, ensenarla, y pueblo.

Bagacaua, pueblo, y punta.

Bagacay, or Liloan, arroyo, pueblo junta, rio, y seno.

Bagadila, monte.

Bagaguimbal, rio.

Bagaisi, isla.

Bagajon, rio.

Bagalanao, rio.

Bagalayag, punta.

Bagalejo, punta, y rio.

Bagamanoc, fondeadero, y pueblo.

Bagambagam, grupo de islas.

Bagambangan, isleta.

Bagambanua, isla.

Baganga, ensenada, fondeadero, pueblo, y rio.

Baganito, ensenada.

Bagasaue, punta.

Bagasicao, junta.

Bagasipal, isla.

Bagasulan, punta.

Bagatao, isla, y punta.

Bagatusan, isla.

Bagauma, rio.

Baglit, punta.

Bagnotan, pueblo.

Bago, ensenala, pueblo, y punta.

Bagombong, punta.

Bagorayrang, punta.

Bagoso, punta.

Bagton, punta. 
Baguan, rancheria.

Bagubaud, punta.

Bagugao, barrio.

Baguigan, pueblo.

Baguio, punta.

Bagulayan, punta.

Bagumbang, arroyo.

Bagumbanuo, isla.

Bagumbayan, villaje.

Bagunbanna, islas.

Bagupantac, punta.

Bagutayoc, punta.

Bahay, punta.

Baheli, rio.

Bahia Honda, bahia.

Bahu, monte.

Baigones, punta.

Bais, ensenada, islas, y puehlo.

Baja, punta.

Baja-Ilanura, isla.

Bajao Gunnong, punta.

Bajuyan, villaje.

Balabac, arrecife, estrecho, isla, monte, y jueblo.

Balabao, punta.

Balacling, punta.

Balactasan, puelilo.

Balad, ensenada, y villeta.

Balada, punta.

Baladigan, punta.

Balading, islas.

Balagui, villeta.

Balaguigan, villaje.

Balaiuac, islotes.

Balalo, puerto, y punta.

Balamban, ensenada, pueblo, y punta.

Balanan, rio.

Balangiga, pueblo, y rio.

Balangona, punta.

Balanguingui, grupo le islas, y isla.

Balangunan, fondeadero.

Balanin, punta.

Balantian, rio.

Balaring, punta.

Balasian, rio.

Balasig, villeta.

Balateros Chico, ensenada.

Balateros Grande, ensenarla.

Balatic, punta.

Balatong, punta.

Balatuan, punta.

Balayan, fondearlero, pueblo, rio, y seno.

Balbagan, isla.
Balcag, rio.

Baldan, bajo.

Baler, rapital, ensenarla, y rio.

Balesin, isla.

Balete, puerto, punta, rio, y seno.

Balianay, isla.

Baliangao, ensenarla, puelso, y punta.

Balibago, punta.

Balicacao, punta.

Balicasag, isla.

Balicuatro, ensenada, islas, y punta.

Baligao, seno.

Baliguian, isla.

Baliguigam, rio.

Balijuagan, rio.

Balimanoc, punta.

Balimbin, Junta, y villaje.

Balinatio, isla.

Balingasag, fonteartero, pueblo, y punta.

Balingauan, punta.

Balingui, isla.

Balino, villeta.

Balintang, canal, y isla.

Baliquias, ensenada.

Baliri, rio.

Baliscan, islote.

Balison, monte, y villeta.

Baliti, ensenada, y puerto.

Balito, rio.

Baliuasan, rio.

Ballantongse, punta.

Ballena, isla.

Balo, rio.

Balogo, punta.

Balolo, punta, y rio.

Balosaro, villaje.

Baloto, puerto.

Baloy, monte.

Balseiro, punta.

Balseiro, (de) canal.

Baltasar, isla.

Baluagan, punta, y rio.

Baluarte, pueblo, y junta.

Balubaruy, seno.

Baluc, punta.

Balucbujan, seno.

Balugo, barrio.

Baluk-baluk, isla.

Balungungan, isleta.

Balunus, rascheria.

Balut, isla.

Balutu, punta.

Balutuan, punta. 
Bamban, punta.

Bambaon, punta.

Bambayan, pueblo.

Bana, punta.

Banacon, isla.

Banago, punta.

Banajao, volean apagado.

Banalacan, or San Andres, puerto.

Banalacan, rio.

Banao, seno.

Banaran, (de) (anal, y isla.

Banate, pueblo.

Banayan, rio.

Banbajon, rio.

Bancal, ensenarla, y punta.

Bancalan, isla.

Bancaobancacn, punta.

Bancolub, villaje.

Bancoran, islas.

Banculin, punta.

Bancungan, isla.

Banda, rillaje.

Banday, villeta.

Bandiloan, junta.

Bando, punti.

Banga, ensenalia, pueblo, puerto, y punta.

Banga-banga, punta.

Bangag, or Rio Chico, rio.

Bangalao, isla.

Bangao, isla.

Bangar, puebli.

Bangilan, rio.

Bangon, villaje.

Bangud, islote.

Bangued, rapital.

Bangui, puelslo, y semo.

Bangun, rio.

Bani, puello, y punta.

Banicaa, rio.

Banigan, fondealero, y junta.

Banilad mayor, lajo.

Banilad menor, bajo.

Banisilan, rio.

Banison, villaje.

Banjan, islote.

Banla, calo, y punta.

Banod or Gazan, punta.

Banon, isla.

Banop, punta.

Baños, punta.

Banot, isleta.

Bantac, isla.

Bantaranan, isla.

Bantay, jueblo.

Bantayan, isla, y pueblo.
Bantigui, isla, y punta.

Bantique, puntil.

Banton, isla, monte, y pueblo.

Bantoncillo, grujo de islas, y isla.

Bantuin, punta.

Bantulin (1) Bantolinao, punta.

Bantulinos, islotes.

Banua, punta.

Banvilan, funti.

Banyao, grupo te islas, y isla.

Banyo, rio.

Bao, punta, y rio.

Baoang, pueblo, y punta.

Baobaon, punta.

Baodicay, rio.

Baqueque, islote.

Baquiao, villaje.

Baquit, lahia.

Bararin, isleta.

Baras, fondeadem, fuerte, pueblo, puerto, punta, y rio.

Barasbaras, punta.

Barat, rio.

Barauan, rio.

Barayan, monte.

Barayon, punta.

Barayong, rio.

Barbacan, sierra, y villaje.

Barbaza, pueblı.

Barcelona, puel,lı.

Bari, isla, y punti.

Barias, bajo.

Barili, ensenada, y puello.

Bariu, isleta.

Baroanga, puet)lo.

Barocay, isleti.

Barotac Nuevo, pueblo.

Barotac Viejo, pueblo, y rio.

Barrabas, bajo.

Barra Laylay, villaje.

Barrera, puerto.

Barton, puerto.

Barubaragna, isla.

Barugo, pueblo, y punta.

Baruro, rio.

Baruyan, rio.

Bary Chico, punta.

Basaan, isla.

Basainga, puerto.

Basbas, (de) canal, fondeadero, isla, punta, y seno.

Basco, villaje.

Basdio, punta.

Basey, fueblo, puerto, y rio.

Bashi, canal, y rocas. 
Basi, pueblo.

Basiao, islas, y monte.

Basiauang, ensenala, y punta.

Basibuli, banco.

Basic, isla.

Basigacon, punta.

Basigajon, punta.

Basilan, estrecho, y islas.

Basin, islas.

Basing, cascalia.

Basirang, arroyo, y punti.

Basol, isla.

Basot, isla.

Bassa, punta.

Bassie, isla.

Batac, jueblo.

Batag, isla.

Batan, or Basay, hajo, cabo, isla, puerto, y punta.

Batanes, grupo de i.las.

Batangas, eapital, ensenarla, provincia, y rio.

Batanluma, boca.

Bataon, ensenada, y villeta.

Batas, isla.

Batauan, pueblo, puerto, y punta.

Batbatan, isla.

Bateria, punta.

Batgongon, grupo de islas.

Batiano, punta.

Batino, pueblo.

Batnon, puerto.

Bato, lago, monte, pueblo, junta, y rio.

Batoan, ensenarla.

Bato-bato, punta.

Batolaqui, bajos.

Batotindoc, punta.

Batucas, puerto.

Batulayol, punta.

Batulinao, punta.

Batunan, isleta.

Baturaue, rio.

Baturrapa, isla.

Bauan, pueblo, punta, rio, y seno.

Baugan, punta.

Bauisa, pueblo.

Baul, isla.

Baulao, villaje.

Bautayan, monte.

Bay, laguna, pueblo, y punta.

Bayaba, rio.

Bayabas, cordillera.
Bayagua, punta.

Bayamban, junta.

Bayarin, punta.

Bayas, islas.

Bayatnan, isla.

Bayauan, puello.

Baybay, monte, pueblo, puerto, punta, rio, y seno.

Baybayon, pueblo.

Bayombong, capital, y ric.

Bayonan, rio.

Bayoria, punta.

Bayugao, villaje.

Belan, isla, y punta.

Belauan, islas.

Belcebu, bajo.

Bello, fondeadero.

Benagalet, punta.

Bentabay, rio.

Bentoan, punta.

Bernacci, monte.

Berrugosa, punta.

Betbet, punta.

Biasong, punti.

Biaui, punta.

Bicobian, puerto.

Bicol, rio.

Bignian, villaje.

Bilaa, punta.

Bilad, punta.

Bilam-bilam, fondeadero.

Bilan-bilan, puerto, y punta.

Bilan-bilangan, isla.

Bilat, arroyo, y punta.

Bilatan, isla, pueblo, y punta.

Biliran, fondeadero, isla, y punta.

Biliran, (de) canal.

Bilitan, grupo de islas.

Billanguan, isla.

Biloan, seno.

Biloc-biloc, banco.

Binabasalan, isla.

Binacayan, rio.

Binadil, ensenarla, y juerto.

Binagmaan, punta.

Binagon, punta.

Binajan, rio.

Binalaba, isleta.

Binalbagan, pueblo, y punta.

Binalio, islote.

Binaliu, rio.

Binan, villaje.

S. Doc. $228-2$ 
* Binanan, isla.

Biñang, puehlo.

Binanga, [merto.

Binanga, or Minangas, ensenarla, puerto, y junta.

Binangonan, fonlearlero, monte, $y$ junta.

Binangtan, islote.

Binantagan, punta.

Binarayan, isla.

Binatac, junta.

Binauan, isia, y serno.

Binauangan, rio.

Binay or Oacan, punta.

Bingay, puntt.

Biniahan, rio.

Binicoian, junta.

Binictigan, rio.

Binigsian, punta.

Biniptican, punta.

Binlana, monte.

Binloc, barra.

Binmacan, monte.

Binobong, arroyo.

Binorungan, punta.

Bintoot, isla.

Bintoulan, isla.

Binuan, rio.

Binuanga, pueblo.

Binuangan, punta.

Binugayan, punta.

Binulbulan, isla.

Binulin, rio.

Binuluangan, isla.

Binunang, punta.

Binuncalan, junta.

Binuni, punta.

Binunsalian, puerto.

Binuntuan, junta.

Biquin, punta.

Biri, isla, y pueblo.

Bisayan, junta.

Bisayas, grupo de islas.

Bislig, ensenada, pueblo, y punta.

Bisucay, isleta.

Bitad, isla.

Bitan, arroyo, y barrio.

Bitanjuan, Irunta.

Bitas, rio.

Bitinan, islas.

Bitinosa, isla.

Bito, laguma, punta, y rio.
Biton, ensenada, y villaje.

Bitoon, pueblo, y punta.

Blanca, punta.

Blik, monte.

Bloqueo, estrecho.

Boac, fondeadero, y rio.

Boalan, villaje.

Boalo, pueblo.

Boasoa, pueblo.

Boay, puertu.

Boaya, punta.

Boayagan, villeta.

Boayan, estero, y isla.

Bobon, pueblo, y punta.

Boca, funta.

Bocaboc, islas, y (de) paso.

Boca Chica, estrecho or jaso.

Boca Grande, paso.

Bocalton, villeta.

Bocator, arroyo, y punta.

Bocboc, or Bantigui, punta.

Bog, punta.

Bogio, punta.

Bogo, punta, y villeta.

Bohol, islas, y monte.

Bojeador, cabo.

Bolaboc, punta.

Bolalo, bahia.

Bolila, punta.

Bolinao, cabo, pueblo, y puerto.

Bolipongpong, islas.

†Boljoon, pueblo, y seno.

Bollonan, punta.

Boloang, islas.

Bolod, islas.

Bolong, fondeadero, y pueblo.

Bolud, punta.

†Bombon, lago, monte, y punta.

Bombonon, puerto, y punta.

Bonat, arroyo.

Bondog, pueblo, y punta.

Bondulan, junta.

Bonete, punta.

Bonga, isla, y monte.

Bongabon, jueblo.

Bongao, canal, islas, y puerto.

Bongo, islas, y junta.

Bonleo, rio.

Bonta, punta.

Bontoc, capital, y distrito.

Bontol, villeta.

Boomboong, isla. 
Borabo, isla.

Borac, pherto.

Borbon, pueblo.

Borobaloto, bajo.

Borobo, rio.

Borocay, isleta.

Boronga, punta.

Borongan, pueblo, puerto, y punta.

Bosicong, arroro.

Bosque, punta.

Botalon, bahia.

Botavanan, isla.

Botic, isla.

Botobolo, punta.

Botogan, punta.

Botolan, monte, pueblo, y punta.

Botong, punta.

Bototinlot, punta.

Bowen, isla.

Briyoon, punta.

Buac, rio.

Buad, canal, y isla.

Buagan, punta.

Buan, islas, y punta.

Buang, seno.

Buanoy, punta.

Buartia, islas.

Buas, punta.

Buasanguay, rio.

Buatpisan, estero.

Buaya, islote.

Bublaran, punta.

Bubon, punta.

Bubuan, islas, y monte.

Bubunauan, rio.

Bucagan, colina.

Bucaland, rio.

Bucao, rio.

Bucas, isla, y monte.

Bucotingol, boca.

Buctun, rio.

Bucud, punta.

Bucutona, isla.

Bucutua, isla.

Bucutuan, grupo ile islas.

Budog, punta, y seno.

Buenabrigo, fondeadero, y punta.

Buenavista, puehlo.

Buenli, ensenarla.

Buen-retiro, villaje.

Bugan, rio.

Bugason, pueblo.

Bugay, villaje.

Bugaya, punta.
Buglug, isla.

Bugnai, arroyo, punta, y senes.

Bugnayon, junta.

Bugnallan, punta.

Bugo, punta.

Bugod, punta, y rillaje.

Bugolibas, rio.

Bugsanga, punta.

Bugsuk, isla.

Bugtason, rio.

Bugton, punta.

Bugtu, punta.

Bugtun, isla.

Bugubas, rio.

Buguey, pueblo, y puerto.

Bugui, isla, y punta.

Buguingui, pherto.

Buguis, punta.

Buguitay, isla.

Bugulayan, punta.

Bugut or Carmen, villeta.

Bugut, puerto.

Buhi, lago, y puelsh.

Buhisan, punta.

Buh-Sulat, bajo.

Bujatan, rio.

Bujimejil, isla.

Buju, islote.

Bula, pueblo, y unta.

Bulabot, rio.

Bulac, rio.

Bulacahui, punti.

Bulacaue, punta.

Bulac-bulac, funta, y villeta.

Bulag, ensenada.

Bulagao, monte.

Bulalacao, ensenada, isli, pueblo, y punta.

Bulalaqui, punta.

Bulaluan, punta.

Bulan, isla, pico, y pueblo.

Bulana, punta.

Bulangolan, punta.

Bulangsi, punta.

Bulanjao, monte.

Bulansa, rio.

Bulato, punta.

Buliasiun, bintay.

Buliculut, pueblo.

Bulicutin, isla.

Buliluat, punta.

Bulilungun, rio.

Buliluyan, cabo.

Bulipunti, aguarla. 
Bulitalun, punta, y rio.

Bulitun, isla.

Bulloc, rio.

Buloc, seno.

Bulot, punta, y rio.

Buluan, isla, y laco.

Bulubadian, isla, y punta.

Bulubadiangan, isla.

Bulubutu, punta.

Bulugisan, punta.

Bulusan, puebio, punta, y volean artivo.

Bulusao, rio.

Bulutuan, punta.

Bunauan, rio.

Bun-bun, pueblo.

Buncalon, punta.

Bunga, punta, y villeter.

Bungas, rio.

Bungcanauay, punta.

Bunglas, rio.

Bungul, punta, y seno.

Bungulbungul, isleta, y villaje.

Bunog-bato, punta.

Bunop, punta.

Bunot, punta, y rio.

Bunot-Pasil, isla.

Bunsali, rio.

Buntud, hajo.

Bunubog, punta.

Bupi, isla.

Bura, punta.

Burabot, punta.

Burac, punta.

Buracay, isla.

Buralman, seno.

Burayot, punta.

Burbadian, seno.

Buri, isla, y punta.

Burias, isla.

Burijan, ensenarla.

Burnay, monte.

Burobodiongan, junta, y seno.

Buruanga, pueblo.

Buruhan, rio.

Burumban, villaje.

Buruncan, punta.

Burungburugan, isla, y monte.

Burutan, isla.

Bury, punta.

Busin, isla, y puerto.

Busluc, punta.

Busuanga, islas, pueblo, punta, y rio.

Busubusug, rancheria.

Busud, rio.
Busunga, irla.

Butag, ensenala, y visita.

Butaira, seno.

Butananan, punta.

Butbilao, monte.

But Gaatan, monte.

Butilan, estero.

Butilao, monte, y puel)lo.

Butlang, isla.

Butpula, monte.

Butuan, bahia, capital, y rio.

Butulan, volcan apagado.

Buyallao, isla, y punta.

Buyo, villeta.

Byan, isla.

Ca-angal-angalan, rio.

Caba, pueblo.

Cabacan, monte, pueblo, y rio.

Cabacbatan, punta.

Cabaccongan, punta.

Cabacsalan, isla, y rio.

Cabacuan, punta.

Cabacungan, colina, punta, y villeta.

Cabadiancan, punta.

Cabagan, punta, y villaje.

Cabagayan, isla.

Cabaglausan, rancheria.

Cabahan, isla.

Cabalac, punta.

Cabalagnan, pueblo, y punta.

Cabalan, rio.

Cabalanan, islote.

Cabalanga, rio.

Cabalas, pico.

Cabalasan, rio.

Cabalata, monte.

Cabalete, isla.

Cabalian, monte, puel,lo, y punta.

Cabaliarian, isla.

Cabalitian, isla.

Caballete, monte.

Caballo, pulo, y punta.

Cabalocan, colinas.

Cabalua, isla.

Cabaluan, rio.

Cabangan, pueblo, y punta.

Cabangsan, villaje.

Cabanian, punta.

Cabano, rio.

Cabantian, punta.

Cabantiguianes, islas.

Cabantocuan, rio.

Cabantuan, isla. 
Cabaquian, isla.

Cabaran, estero.

Cabaranan, ensenarla, y funta.

Cabarasan, punta.

Cabarruyan, isla.

Cabarsana, rio.

Cabasbatan, punta.

Cabauan Grande, isla.

Cabaun, isla.

Cabayan, pueblo, y punta.

Cabcaban, rio.

Cabcabe, puntil.

Cabeza, punta.

Cabeza de Bondog, monte.

Cabeza de Negrito, (de) paso.

Cabezo de Tablas, monte.

Cabgan, isla, y punta.

Cabiangon, rio.

Cabiao, pueblo.

Cabibijaban or Vinas, rio.

Cabicungan, punta, y rio.

Cabidian, rio.

Cabilan, isla.

Cabilao, isla.

Cabilauan, isla.

Cabilonan, punta.

Cabiluan, isleta.

Cabingaan, isla.

Cabitoagan, rancheria.

Cablagna, punta.

Cabodiangan, punta.

Cabog, punta.

Cabolijan, hentay, y rio.

Cabong, rio.

Cabongon, rio.

Cabra, islas, y (de) paro.

Cabuayan, rio.

Cabucan, isla.

Cabug, punta.

Cabugaan, isla.

Cabugabuan, punta.

Cabugan, isla.

Cabugao, ensenarla, isla, puej)lo, y punta.

Cabugauan, punta.

Cabugayan, rio, villeta.

Cabujan, seno.

Cabulan, isla.

Cabulauan, or Sombrero, isla.

Cabuli, isla, y punta.

Cabulic, punta.

Cabulig, fondeadero.

Cabuluang, rio, y seno.

Cabulung, rio, y seno.

Cabunan, isla.

Cabunganan, punta.
Cabungeut, seno.

Cabungeoen, islas.

Cabungian, lantay.

Cabungoan, isla.

Cabuntog, puebl'r.

Cabura, punta.

Caburan, fondeadero.

Cabusa, punta.

Cabusao, pueblo, y rio.

Cabut, isla.

Cabuyoc, punta.

Cacamatacan, punta.

Cacataan, islas.

Cacayatan, isleta.

Cacbalisay, isla.

Cacbolo, isleta.

Cacbucao, isleta.

Cacinjia, islas.

Cacnipa, isleta.

Cacraray, isla, (de) paso, y punta.

Cacub, pueblo.

Cadac-an, rio.

Cadburauan, wr Panganiran, punta.

Cadagun, lago.

Caddanat, rio.

Cadiz Nuevo, pueblo, y riu.

Cadlao, isla.

Cadnaan, villeta.

Cado-ocan, punta.

Caduayan, pueblo.

Caducdula, punta.

Cadulan, rio.

Caduruan, pueblo, y punta.

Cagan, or Banga, punta.

Cagayan, fondeadero, isla, laguna, puetslo, y rio.

Cagayancillo, islas.

Cagayanes, islas.

Cagbalisay, isla, y punta.

Cagbanlio, isla.

Cagdanaon, isleta.

Cagdullon, islotes.

Caglanipan, arroyo.

Cagog, punta.

Cagoncon, rio.

Cagpoc, punta.

Cagpog, punta.

Caguan, elsenarla, y rio.

Caguayan, puerto, y punta.

Caguco, monte.

Caguipot, isla.

Caguiuan, punta.

Caguiuian, arrovo.

Caguma, punta. 
Caguray, pminti.

Cahayagan, islil.

Caibabo, punta.

Caibiran, monte, pueinlo, y rio.

Caibunuan, punta.

Caicayan, rio.

Caiguit, rio.

Caiholo, rin, y semo.

Caima, ensenark.

Caiman, (del) bahia, bajo, y punta.

Caimanes, or Libungan, (de los) rio.

Cainguin, punta.

Cainta, pueblo, y rio.

Cairaman, rio.

Cairauan, plunta, y rio.

Cairilao, monte.

Caisabo, rio.

Caitalang, monte.

*Cajagnaan, puehlo, y seno.

Cajidiocan, pueblo, y punta.

Cajoagan, isla.

Calaan, punta.

Calabadian, isleti.

Calabago, punta.

Calaban, isla.

Calabanga, pueblo, y rio.

Calabanijan, monte.

Calabaza, punta.

Calabazas, isla.

Calabuctungan, islotes.

Calaca, pueblo.

Calacala, isleta.

Calaclan, punta, y rio.

Caladan, punta.

Caladman, punta.

Calagauan, punta.

Calagua, isla, y pueblo.

Calaguaguin, fondealero, y seno.

†Calaguan, isla, punta, y semo.

Calaguas, islas.

Calaguian, isla.

Calagundian, rio, y villeta.

Calaitan, canales, y isla.

Calaitang, canal.

Calajit, isla.

Calala, monte.

Calalanag, isla.

Calamba, pueblo.

Calambayanga, isla.

Calamian, islas.
Calamianes, grupo de islas.

Calamisan, rio.

Calanatan, monte.

Calancan, ensenata, y rio.

Calandanun, estero.

Calandorang, bahia.

Calangaman, isla.

Calangunan, estero.

Calanhayaun, isla.

†alaocalao, punta.

Calapacuan, punta.

SCalapan, capital, ensenada, y punta.

Calape, isla, pueblo, y puerto.

Calapocan, punta.

Calatan, monte, y punta.

Calatayan, punta.

Calaton, ensenada, monte, y punta.

Calatrava, pueblo.

Calauagan, punta.

Calauang, punta.

Calaui, punta.

Calauit, punta.

Calavera, ensenala.

Calavite, cabo, y monte.

Calayan, fondeadero, islas, y pueblo.

Calayanan, junta.

Calayo or Fuego, junta.

Calbalete, isla.

Calbasag, rio.

Calbayoc, ensenarla, fonteadero, pueblo, y rio.

Calbayogos, punta.

Calbiga, pueblo, y rio.

Calbugan, rancheria.

Calcal, villaje.

Calcuera, punta.

Calcut, punta.

Caldera, ensenala, puerto, y punta.

Calero, punta.

Calian, punta.

Calianan, bajo.

Caliban, punta.

Calibon, punta.

Calibugon, monte.

Calibuyo, torre.

Caligahan, seno.

Caligangang, punta.

Calilidan, punta.

Calinton, isla.

Calipapan, punta. 
Calipipit, isleta.

Calirujan, punta.

Calis, punta.

Caliselan, rio.

Calitan, isleta, y junta.

Calituban, isla.

Caliuauan, punta.

Calivo, pueblo.

Callibugon, monte.

Calmang, punta.

Caloag, barrio, y villaje.

Caloan, rio.

Calocot, isla.

Calog, punta.

Calogangan, punta.

Calomatan, rio.

Calsiguaran, isla.

Caltom, puerto.

Calua, isla.

Caluan, rio.

Caluay, isla, y villeta.

Calubi, punta.

*Calublu, grupo de islas.

Caluccub, villeta.

Calugtang, grupo de islas.

Calugupan, villeta.

Caluman, isla.

Calumbagan, grupo de islas.

Calumbuyan, isleta.

Calumpan, monte, y punta.

Calumpijan, isla.

Calumpit, pueblo.

Calungan, punta, y rio.

Calupag, islas.

Calupan, punta.

Calusa, isla.

Calusian, pueblo, y rio.

Calutan, islote, y villeta.

Caluya, islas, y punta.

Caluyajan, monte.

Calvario, monte, y villaje.

Calver, punta.

Calvija, lago.

Camago, isleta.

Camainsi, punta.

Camalaniugan, pueblo.

Camalian, isla, y punta.

Camalig, punta.

Camamauan, punta.

Camamot, isla.

Camanchito, rio.

Camandag, isla.
Camanga, isla.

Camanhala, or Macoto, ('nsenala.

Camanoc, punta.

Camansi, junta.

Camansusu, punta.

Camaris, ensenala, y punta.

Camasusu, isla, fico, punta, y seno.

Camayan, puntil.

†Cambacamba, (1le) canal.

Cambajao, punta.

Cambalaguio, punta, y ris.

Cambalalon, punta.

Cambalot, bajo.

Cambanog, punta.

Cambarugan, piedras.

Cambari, isleta.

Cambasag, isla, y pueblo.

Cambasingan, islote.

Cambijan, punta.

Cambista, punta.

Cambulayan, punta, y rio.

Cambutatay, rio, seno, y villeta.

Cambutayan, senn.

Cameran, isla.

Camicamian, isla.

Camiguin, isla, y monte.

Camiling, rio.

Camiris, ensenada, y punta.

Camomoy, monte.

Camoropudan, islotes.

Camotes, islas.

Campamento, puntil.

Campao, punta.

Campatoc, puelolo, y punta.

Campilipa, punta.

Campinayucan, villaje.

Campomanes, ensenata.

Campoocan, (de) picos.

Campopo, monte, y seno.

Camu, punta.

Camungyan, inlas.

Cana, estero.

Canaba, punta.

Canabayon, isla.

Canabungan, isla.

Canacana, punta.

Canacao, ensenada.

Canagaayan, punta.

Canaguan, isla.

Canaguallon, islotes.

Canaguayan, islote, phert's, punta, y rio.

Canahauan, islas. 
Canahauan-Daco, islas.

Canahauan-Gutiay, grupo de islas.

Canal Principal, or Gran Canal, canal. Canamay, punta.

Cananay, islote.

Cananyong-Daco, punta.

Canaon, puerto.

Canapuc, punta.

Canaren, lago, pueblo, y rio.

Canaron, grupo de islas.

Cañas, isla, (de) punta, rio, y seno.

Canastilla, (le la) monte.

Canatonatoan, monte.

Canauayan, punta, y villeta.

Canauayor, isla.

Canay, puerto, y villaje.

Cancabato, ensenada.

Cazcrugan, seno.

Cancuman, isla.

Candaba, lago, y pueblo.

Candanaran, isla.

Candaraman, isla.

Candaya, punta.

Candea, puelslo, y punta.

Candian, rio.

Candoaga, rio.

Candolon, punta.

Candolu, isla.

Candon, pueblo, y punta.

Candongos, bajo.

Cangan, junta.

Cangaranan, ris.

Cangat, punta.

Canglonbog, punta.

* Cangouac, punta.

Canicabuyan, isla.

Canigao, isla.

Canigaran, isla.

Caninao, isla.

Caninoan, isla.

Canipan, ensenatia.

Canipo, bahia, islas, y rio.

Can-itong, ensenadia.

Canjalon, junta, y rio.

Canlaon, or Malaspina, volean activo.

Canliling, rio.

Canloay, punta.

Canlongcaniong, arroyo, y sellu.

Canmahana, ensenala.

Canmamot, isla.

Canmanoc, monte.

Canoan, ensenadi, y juerto.

Canomalag, punta.
Canompit, puntia.

Canotoc, punta.

Cansapal, punta.

Cansilan, junta.

Cansumala, punta.

Cantagay, punta.

Cantican, isla.

Cantilan, pueblo.

Cantingas, punta.

Cantlalig, puntia.

Canton, isla.

Cantoto, punta.

Cantutuy, punta.

Canumay, punta.

Canumbao, punta.

Cap, islas.

Capalis, rio, y seno.

Capalonga, pueblo, y rio.

Capanaupuyan, punta.

Capandan, punta.

Capap, ensenata.

Capines, punta, y renu.

Capinitan, rio.

Capis, rio.

Capitancillo, isla.

Capiz, capital, ensenarla, y fomlearleru.

Capnoyan, islas.

Capoas, monte.

Capocpocanan, isla.

Capones, islas, y punta.

Capoocan, puetlo.

Capsalay, isleta.

Capsalon, isleta.

Capsuegan, monte.

Capual, isla.

Capucan, rio, y villeta.

Capul, islas, y punta.

Capulaan, ensenadia.

Capuluan, punta.

Capulujan, monte.

Capunuypugan, punta.

Capuy, villaje.

Caraballo, rio.

Caraballo de Baler, monte.

Carabao, or Carao, ensenarla, isla, y punta.

Carabdapal, junta.

Carabuca, punta.

Carachan, punta.

Caraga, ensenala, lago, puehlo, y rio.

Caraiman, villeta.

Caramatan, punta.

†Caramuan, monte, puelilo, y punta. 
Caramuran, ensenarla, y rio.

Caranan, ric.

Carancaran, villaje.

Carandaga, isleta.

Carangac, punta.

Cara.sgdalto, punta.

Caranhan, punta.

Caranisan, punta.

Carao, punta.

Carao, or Carabao, ensenada, isla, y punta.

Carapdapat, punta.

Caray-caray, rio.

Carcar, puerto, punta, y rio.

Careat, villaje.

Carigan, punta.

Carigara, pueblo, ! seno.

Caringo, isla.

Caritan, pueblo.

Carlaga, rio.

Carles, pueblo.

Carmelo, punta.

Carmen, hanco, y pueblo.

Carmencito, bajo.

Carmina, pueblo.

Carmona, isla, y pueblu.

Carnasa, isla.

Carogo, isla.

Caromata, monte, puel)lo, y' seno.

Carongdong, puebla.

Carranglan, pueblo, y rio.

Cartagena, ensenada, y puelolo.

Casanalan, bantay.

Casangayan, punta.

Casauman, laguna, y punta.

Cascada, punta, y rio.

Casignan, rio.

Casiguran, ensenada, jueblo, y rio.

Casilagan, punta.

Casilaran, seno.

Casilian or Badoc, rio.

Casirahan, isleta.

Casolgan, paso.

Castilla, puerto.

Castillo, (del) punta.

Casuarina, punta.

Casul, seno.

Casulian, isla.

Casumalac, punta.

Cataaba, puerto.

Catabanga, punta, y rio.

Catabauan, ensenadi.

Catablas, rio.
Catabunan, isla.

Catadinan, punta.

Catadman, junta, y rillaje.

Catagbacan, encenarla, y rio.

Cataingan, puehlo, y puerto.

Cataisan, punta.

Catala, monte, y punta.

Catalaban, islit.

Catalangan, rio.

Catalo, punta.

Cataluan, punta.

Catanauan, ensenada, y juehlı.

Catanduanes, isla.

Catanduculan, seno.

Catanungan, punta.

Catao, punta.

Cataringting, punta.

Catarman, fondeatero, pueblo, purto.

y punta.

Catbalogan, eapital, y fomlealero.

Catbatan, punta.

Catdun, punta.

Catel, pueblo, punta, y rio.

Catigtuan, villaje.

Catimag, ensenarla.

Catimu, jumta.

Catinic, punta.

Catiyoman, punta.

Catmon, arroyo, monte, pueblo, punta, seno, y rio.

Cato, baluarte.

Catoan, punta.

Catolog, pueblo.

Catpatin, isla, y punta.

Catuaba, puerto.

Catuisan, punta.

Caua, rio, y volean activo.

Cauampit, punta.

* Caunhalan, punta.

Cauayan, islas, pueblo, puerto, punta, y rio.

Cauayanen, isleta.

Caucane, punta.

Cauit, barra, ensenada, pueblo, puerto, punta, rio, y reno.

Cauitan, punta.

Caunaagan, punta.

Caunpan, puehlo.

Caurusan, or Siruma, punta.

Causilan, punta.

Cavantiguianes, islas.

Cavilli, or Caueli, islas. 
Cavite, puerto.

Cavite Viejo, pueblo.

Caxisigan, isla.

Cayangon, punta.

Caybiran, puista.

Caygan, islote.

Cayope, rio.

Cayuagan, islit.

Cazador, punta.

Cazadores, cabo.

Cazcarro, islotes.

Cebu, capital, (de) estrerho, fomlealero, $y$ islas.

Centinela, punta.

Cerro, (del) punta.

Cervera, pueblo, y punta.

Cetaceo, monte.

Cheron, isleta.

Chinampan, caho.

Chinayan, pico.

Chindonan, isla.

Chinicaran, isleta.

Chocolate, isla.

Chongos, ensenada.

Cidad, villeta.

Ciego, puerto.

Cien (Las), fondearlero, y islas.

Cilancan, puelslo.

Cinapuran, grupo de islas.

Clan, rio.

Clarendon, balia.

Claudio, punta.

Claveria, "Cabicungan, puebl».

Coambug, rancheria.

Coamen, isla.

Cobrador, islas.

Cobulamang, estero.

Cocaguayan, punta.

Cocales, punta.

Cochinos (Los), islas.

Cocina, lago.

Coco, isla.

Cococ, isla.

Coconongon, punta.

Cocoro, islas.

Codiapi, monte.

Codon, pueblo, punti, y rio.

Cogon, punta.

Cogonal, pico.

Cogoncogon, punta.

Colan, colina.

Colapian, punta.

Colapsin, punta.
Colasi, pueblo, punta, rio, y sierra.

Colindo, estero.

Coloby, hajo.

Coloconto, seno.

Colocoto, rocas.

Coloma, bajo.

Colorada, or Norte, punta.

Comas, isla.

Cominauet, punta.

Comiran, (de) canal, y isli.

Comocutuan, isleta.

Compostela, pueblo.

Conas, seno.

Concas, banco.

Concepcion, capital, y (de la) punta.

Concongon, punta.

Conglonbog, punta.

Conico, monte.

Conimo, rio.

Cono, grupo de islas, y monte.

Consumala, punta.

Copia, fondeatero, $y$ isla.

Copton, monte, pueblo, punta, y seno.

Coral, bahia.

Corandagos, islas.

Corcuera, fuerte, golfo, isla, y pueblo.

Cordelia, punta.

Coroan, rio.

Corocolapot, seno.

Coromata, seno.

Coron, (de) estrecho, golfo, isla, y pueblo.

Coronado, bahia, y punta.

Coroncoron, arroyo, punta, y seno.

Corregidor, isla, y puerto.

Corte, monte, pueblo, y punta.

Cortes, colina, y pueblo.

Coruan, puerto, y punta.

Corumi, monte.

Costanera, punta.

Cota, fuerte.

Cotabato, capital, distrito, y fondearlero.

*Cotcot, punta, y rio.

Cotteral, punta.

Cran, or Eran, bahia.

Cresta, isla, y monte.

Cruz, punta.

Cruz de Mayo, bahia.

Cuadrado, islote, y monte.

Cuatro, islas.

Cubabo, punta.

Cubcub, isla.

Cubijan, isla.

Cuby, punta. 
Cucurrayan, isleta.

Cudao, islote.

Cudarangan, (de) colina.

Cudtingan, monte.

Cuernos de Negro, monte.

Cueva, punta.

Cujangal, isla.

Cujangan, isla.

Cujao, punta.

Culaba, villeta.

Culaman, rio.

Culasi, ensenarla, monte. funta, y villaje.

Culasian, rada.

Culebra, isla.

Culili, monte, y punta.

Culion, ikla, monte, pueblo, y puerto.

Cullun, punta.

Cumalarang, rio.

Cumbre, monte.

Cunilan, isla.

Cupiat, isla.

Curo Coayan, islotes.

Currimao, ensenarla, y puerto.

Cutipanan, isla.

Cuyacub, punta.

Cuyo, capital, y isla.

Cuyos, giupo de islas.

Dabun, islote.

Daca, punta.

Daco, isla.

Dacula, isla.

Dado, isleta.

Daet, pueblo, y rio.

Dagdagun, punta.

Dagdap, punta.

Daguet, punta.

Daguinsara, puerto.

Dagun, lago.

Dagupan, pueblo, y puerto.

Daigajon, punta.

Daijagon, ensenada, punta, y rio.

Daimpaquit, punta.

Daingapic, punta.

Daja, villeta.

Daja-Daco, punta.

Daja-Diotay, punta.

Dijican, punta.

Dajugan, grupo de islas.

Dajuntajun, isla.

Dalaga, punta.

Dalagnan, rio.

Dalaguete, pueblo, y punta.

Dalana, estero.
Dalanao, rio.

Dalanas, rio.

Dalanganem, isleta.

Dalauan, bahia.

Dalauana, punta.

Daliaun, bajo.

Dalipe, punta.

Dalit, punta.

Daljugan, punta.

Dallana, rio.

Dalugdug, punta.

Daluman, isla.

Dalupiri, isla, y villeta.

Damacan, punta.

Damao, isleta.

Dambalasan, estero.

Damita, islote.

Dammi, islas.

Damocan, isla.

Damunil, rio.

Danabasot, punta.

Danabusot, punta.

Danajon, Janeo, y isla.

Danao, monte, puehlo, punta, y rio.

Danaodanauan, isla.

Dancalan, pueblo, y punta.

Dancan, punta.

Dandugan, punta.

Dangal, punta.

Danim, punta.

Danjugan, isla.

Danlig, pueblo.

Dansalan, estero.

Dao, monte, pueblo, y rio.

Dapa, isla, (de) paso, y pueblo.

Dapdap, pueblo, punta, y rio.

Dapitan, bahia, capital, puerto, rio, y seno.

Dapuan, villaje.

Dapulisan, punta.

Daquio, isla.

Daquit, punta.

Daquitdaquit, islote, y pierlra.

Daracotan, bahia, islu, y seno.

Daraga, pueblo, y rio.

Darajuay, islas.

Daram, canal, isla, y monte.

Dardara, barrio.

Darigayos, pueblo, y punta.

Darimibo, isla.

Daromaoang, punta, y rio.

Darsena, isleta, y junta.

Darumauan, rio.

Dasaan, isla. 
Dasol, fondearlero, futhlo, y. seno.

Dassalan, isla.

Datinuaua, punta.

Datto Ara, rancheria.

Datto Macud, estero.

Datto Mamon, rancheria.

Datto Panguliman, rancheria.

Datu Bato, isla.

Dauan, isla.

Dauigan, ensenarla. y punta.

Dauilican, villaje.

Dauin, pueblo.

Dauis, uneblo.

Dauit, punta.

Daul, rio.

Dauli, finta.

Davao, capital, fondeadero, rio, y seno.

Dawil-dawil, isla.

Dayagon, rilleta.

Dayana, isla y punta.

Dayap, ensenala, y punta.

Deagan, islas.

Deato Bato, isla.

Decepcion, punta.

Delgada, runtat.

Delian, islas.

Del Medio, islas.

Deoto-Bata, isla.

Dequez or Goat, islas.

Deseada, puntaz.

Desgraciada, punta.

Desolacion, punta.

Destacada, isla.

Destacado, hajo, y haneo.

Diablo, paro, y (lel) punta.

Diagao, punta.

Dialao, punta.

Diamante, lajo.

Dianglit, isleta.

Dibanca, isleta.

Dibatuc, isleta.

Dibayabay, punta, y rio.

Dibinisa, punta.

Dibinubo, isla.

Diboyoyan, isla.

Dibugha, isla.

Dibuluan, isleta.

Dibut, ensenatia.

Dicabaito, isla.

Dicalabian, isleta.

Dicalabuan, punta.

Dicalaum, isleta.

Dicalayo, villaje.

Dicapen, riv.
Dicapilarin, junta.

Dicapinisan, junta.

Dicapululan, isla.

Dicbilen, pieilra.

Dicoyan, isleta.

Didica, rolcan activo.

Diente, colina, y (del) punta.

Digosan, riı.

Diguilingan, isleta.

Dila, rio.

Dilasac, labia, ensenaila, y puerto.

Dile, punta.

Dimalansan, puerto.

Dimalooc, arroyo, y sello.

Dimancal, isla.

Dimanglet, isli.

Dimaquiat, isla.

Dimiao, pueblo.

Dimipac, isla.

Dimpao, punta.

Dinacsaan, punta.

Dinagat, capital, y isla.

Dinagsaan, punta.

Dinapiqui, punta.

Dinaran, isla.

Dinas, villaje.

Dinatadmo, punta.

Dingalan, Jahia, ensenada, y rillaje.

Dinigman, rio.

Dinuculan, punta.

Dio, islote.

Diogo, or Redonda, islas.

Diotoring, isla, y punta.

Dipaculao, villaje.

Dipalian, isleta.

Dipeldet, isleta.

Dipolod, islas.

Dipolog, pueblo, y rio.

Dippolot, isla.

Dipulao, eneenalia wherto.

Dipulut, isla.

Dirique, ensenada, $y$ foncleadero.

Disabungan, rio.

Disaster, - calor.

Disiasi, punta.

Distoring, islote, y punt:.

Disumangit, junta.

Dit, islit.

Ditacop, isleta.

Ditali, rillaje.

Ditaytayan, isletia.

Ditnot, isleta.

Ditorian, islas.

Ditulay, riv. 
Diualan, punta.

Diuata, monte, y punta.

Diur, punta.

Diuyu, rio.

Divalais, punti.

Divilacan, seno.

Divinubo, isla.

Diviuisa, puntr.

Doble, isla.

Doc Can, isla.

Dolong Cauit, punta.

Dolores, punta.

Domonclon, ensenarla.

Don, islas.

Donao, pueblo.

Donauan, isla.

Donauang, isla, y punta.

Dondonay, isla.

Dongal, junta.

Dongdong, isla.

Dongon, ensenala, punta, y rio.

Donjon, isla.

Donsalan, rio.

Donsol, pueblo, y rio.

Doog, punta.

Doorly, banco.

Dos Amigos, puerto.

Dos Cuernos, monte.

Dos Hermanas, islas.

Dos Picos, monte.

Dragon, punta.

Dromedario, monte.

Ducabaita, isla.

Duero, pueblo.

Duglio, punta.

Duglun, pueblo.

Dugolaan, punta.

Dulag, pueblo.

Dulaiv, rio.

Dulama, rio.

Duljo, punta.

Duljugan, punta, y rio.

Dulunguin, punta.

Dumagas, rio.

Dumaguete, eapital, y punta.

Dumalagui, rio.

Dumalaylay, rio.

Dumali, monte, $y$ punta.

Dumalin, punta.

Dumangas, bantay, estero, y punta.

* Dumanjug, pueblo, y punta.

Dumanquilas, pueblo, punta, y seno.

Dumaquit, punta.
Dumaran, balia, (de) canal, fondeadero, isla, y pueblo.

Dumini, seno.

Dumulug, rio.

Dumunpalit, isla.

Dumuru, rio.

Dumurug, punta.

Dunao, isla.

Dunaun, isla.

Duncalan, villaje.

Dung, fonlearlero.

Dungun, punta y villaje.

Dunol, ensenarla.

Dunurot, ric.

Dupon, bahia, punta, y ris.

Dupulisan, punta.

Duquino, isla.

Duquis, pueblo.

Durudeen, punta.

Durundeen, punta.

Duruungan, rio.

Duyagan, punta.

Elcano, bajo.

Elefante, isla.

Elet, isla.

Ellis, banco.

El Pardo, pueblo.

El Real, villaje.

Emelina, isla.

Emergencia, punta.

Emmit, punta.

Encanto, punta.

Encarnada, punta.

Engaño, cabo.

Engañoso, monte.

Eniaran, isleta.

Enmedio, hajos, y (de) canal.

Enrile, pueblo.

Entalula, isleta.

Entilan, punta.

Eran, or Cran, bahia.

Erenas, seno, y villeta.

Ermita, (La) pueblo.

Escalante, pueblo.

Escarceo, punta.

Escarpada, isla, y punta.

Esconchada, punta.

Escucha, isla.

Eseo, bajo.

Esfuerzo, (del) punta.

España, bajo, pueblo, y rio.

Espina, punta. 
Espiritu Santo, cabo.

Estancia, fondeadero.

Estilete, monte.

Eulalia, villeta.

Eulili, punta.

Eustasia, bantay, y punta.

Faltabin, isla.

Famosa, villeta.

Farol, isla, y punta.

Faunil, estero.

Fernandez, punta.

Filantropia, punta.

Flaca, punta.

Flechas, punta.

Font, isla.

Foon-Pandanan, punta.

Fortun, isla.

Fragata, rio.

Fraile, isla.

Frailes, islas.

Fuego (de), or de Calayo, punta.

Fuga, islas.

Fulin, isla.

Gaba, seno.

Gabo, puerto, y junta.

Gabot, punta.

Gabung, isla.

Gadd, roca.

Gajo, punta.

Galangalan, monte.

Galban, or Malagundi, punta.

Galera, puerto, y punta.

Gallina ( $\mathrm{La}$ ) y Los Pollos, islas.

Gallo (Cresta de), isla, ! monte.

Galoc, islas.

Galvaney, isla, y punta.

Gam, punta.

Gamay, punta.

Gan, seno.

Gandol, isla.

Ganga, punta.

Ganon, pueblo.

Ganoy, lago.

Gantung, monte.

Gapan, rio.

Gapay, rio.

Gapo, (de) paso.

Gapus, monte.

Garan, punta.

Garcia, baje.

Gardiner, isla.

Gardoqui, punta.

Garot, rio.
Garza, isla.

Gasacan, punta.

Gasan, pueblo, y monte.

Gaspar, isla.

Gata, or Bulag, punta.

Gatas, isla.

Gato, islote.

Gaus, isla.

Gavet, arroyo, y seno.

Gavilan, punta.

Gay, isla.

Gayo-mayo, canal.

Gazan, or Bano, punta.

Geladioc, rio.

Geminis, islotes.

General, isla.

Genuruan, isla.

Getafe, pneblo.

Gibong, rio.

Gibuson, isla.

Gigantangan, isla.

Gigantes, islas.

Gigoso, punta.

Gilutugan, isla.

Gim, rio.

Gimalic, punta.

Gimanoc, isla.

Ginablan, punta, y visita.

Ginac, ensenada.

Ginatilan, pueblo.

Ginigaran, pueblo, y rio.

Ginoso, visita.

Gintacor, cerro.

Ginunagan, pueblo.

Gipdo, isla.

Gitana, isla.

Glan, villeta.

Glan-Masila, fondeadero.

Gnat, arrecife.

God, isla.

Gogo, punta.

Goitya, bajo.

Golgotha, cabo.

Golo, islas, y (cle) paso.

Gomagamot, arroyo.

Gomez, or Lalangan, ensenada, y punta.

Gondra, islote.

Gorda, punta.

Gordag, punta.

Gordu, punta.

Govenen, isla.

Grande, isla.

Grande de la Pampanga, rio.

Granja, villeta.

Gran Laja, isla. 
Guadalupe, villaje.

Guagadi, rio.

Gual, punta.

Guango, punta.

Guanguan, punta.

Guay, ensenada, y punta.

Gubat, ensenada, y pueblo.

Guibauan, or Lamutun, monte, y pueblo.

Guidadalao, punta.

Guigaamon, punta.

Guihalinan, punta.

Guijulugan, pueblo, y rio.

Guilbay, punta.

Guilong, villaje.

Guilutugan, isla.

Guimanan, isieta.

Guimaras, isla.

Guimba, seno.

Guimbal, pueblo.

Guimugahan, punta.

Guinabaranan, punta.

Guinabasan, rio.

Guinabasan, or Buenobriga, punta.

Guinad, punta.

Guinagdong, rio, y punta.

Guinaguanan, ensenada.

Guinanug, punta.

Guinapac, rocas.

Guinaran, rio.

Guinasayan, rio.

Guinauayan, isla.

Guinayan, bantay, y puerto.

Guincapatdan, punta.

Guindabdaban, isleta.

Guindanan, punta.

Guinduganan, punta.

Guindulman, ensenada, monte, preblo, $y$ rio.

Guinhalinan, punta.

Guinigaran, villaje.

Guinitgan, punta.

Guinlabagan, isla.

Guinlabo, islas.

Guinlanagan, pueblo.

Guinlantuyan, punta.

Guinlep, isleta.

Guinlepen, isleta.

Guinluthagan, isla.

Guinobatan, pueblo, y rio.

Guinojoan, punta.

Guinpatayan, punta.

Guintacan, isla, y punta.

Guintiguian, rio.

Guintin, punta.
Guintinguron, rio.

Guintinua, isla.

Guintulan, punta.

Guintungauan, isleta, y punta.

Guion, rio, y visita.

Guirayan, monte.

Guisijan, puełlo.

Guitacor, monte.

Guitinguitin, $\ldots$ Sibuyan, monte.

Guiuan, isla, y puehlo.

Guiuanon, isla, y punta.

Gujangan, isla.

Gulayan, rio.

Gumalac, isla.

Gumay, ensenada.

Gumilan, isla.

Guntao, isla.

Guntuan, isla.

Gusaran, rio.

Gusu, rio.

Guyang, isla.

Habichuela, isleta.

Hagonoy, laguna, y pueblo.

Halaba, punta.

Halcon, monte, y roca.

Hama-hamaang, isla.

Hamurauan, bajo, y junta.

Hassaan, villaje.

Havoley, isla.

Hegad, isla.

Hermana Mayor, isla.

Hermana Menor, or Macalira, i-la

Hermanas, islas.

Hermanos, islotes.

Hernani, pueblo.

Hiabong, pueblo.

Hibago, villaje.

Hibapsan, isla.

Hibog, rio.

Higad, islas.

Higanligam, punta.

Higasaan, rio.

Higunum, isla.

Higununun, i-la.

Hijo, rio.

Hilaban, isla.

Hilongos, pueblo, y rio.

Himamaylan, pueblo, y rio.

Himbucgan, punta.

Himoto, rio.

Himuquitan, isla.

Hinamoc, isla.

Hinatuan, or Ginatuan, or Jinatuan, isla, y pueblo. 
Hinauligan, punta.

Hindang, jueblo.

Hingantingal, rio.

Hingatungan, punta.

Hinigaran, rio.

Hinondayan, seno.

Hinunangan, ensenarla, y pueblo.

Hinundayan, puelslo.

Hinunucan, punta.

Hipudios, villaje.

Hirapsan, isla.

Hirigatungan, punta.

Hitumnog, punta.

Hoangon, punta.

Homonhon, or Jomonjol, or Malhon, isla.

Honda, bahia, punta, y rio.

Horada, isla, y punta.

Hornos, punta.

Hoya de Iba, fondearlero, y punta.

Hubasan, pueblo.

Humangan, punta.

Hurao, or Curao, monte.

Iba, capital, fonclealero, monte, y punta.

Ibachin, isleta.

Ibaicay, punta.

Ibajay, pueblo, punta, y rio.

Ibalong, punta.

Iban, isla.

Ibay, pueblo.

Ibayat, isla, y monte.

Ibelbel, isleta.

Ibugos, or Bashi, isla.

Ibulao, rio.

Ibus, isla.

Icadambanauan, islas.

Icauayan, punta.

Igan, isla, y seno.

Igang, punta.

Igasan, punta.

Igat, seno.

Iglesia, (le la) punta.

Iglesia de Bagombayan, villaje.

Iguana, banco.

Iguic, pueblı.

Iguicon, grupo le islas.

Ilaba, punta.

Ilang-ilang, rio.

Ilaya, pueblo, y rio.

Ilian, villeta.

Iligan, bahia, pueblo, y punta.

Ilijan, colina, ensenada, y punta.

Ilin, monte, isla, pueblo, y punta.

Ilinid, punta.

Ilinin, punta.
Illana, (de la) bahia.

Iloc, estero, y isla.

Iloilo, eapital, (de) estero, estrecho, provincia, puerto, y rio.

Imalagun, isleta.

Imapoto, villaje.

Imaruan, isla.

Imuruan, bahia, fondeadero, y isleta.

Imus, pueblo, y rio.

Inabonga, rio.

Inabuyatan, isla.

Inacauan, punta.

Inaclian, punta.

Inagayan, seno.

Inagbuan, punta.

Inaguican, punta.

Inalauan, rio.

Inambuyod, isleta.

Inamoc, barrio.

Inampulugan, isla.

Inantan, rio.

Inapupan, isleta.

Inararan, punta, rio, y visita.

Inarijan, rio.

Inasang, pico.

Inausugan, seno.

Inautan, villeta.

Inayauan, pueblo, y punta.

Indagamy, isleta.

Indagau, isla.

Ingalan, isla.

Iniguian, arroyo, $y$ punta.

Initao, pueblo, y punta.

Initong, monte.

Iniyao, islote.

Inlulutoc, Jahia.

Inog, seno.

Inogbun, villaje.

Inopacan, puello, y seno.

Iot, puntir.

Ipayo, punta.

Ipayoc, hahia, y rio.

Ipil, bahia, monte, pueblo, purta, y rio.

Ipolote, ensenala.

Iponan, pueblo, y punta.

Iquicon, isla.

Irada, monte.

Iranzo, punta.

Irao, or Culebra, isla.

Iriga, monte, y pueblo.

Iriron, ensenada, pueblo, y roca.

Irong-Irong, punta.

Irraya, monte.

Isabel, punta. 
Isabela, (le la ) paso, provineia, y yeblo. Isabela de Basilan, eapital.

Isac, islote.

Isarog, volcan apagarlo.

Isio, pueblo.

Isioc, punta.

Isla Verde, bahia, y (de) pasos.

Islas Verdes, (le) bahia.

Italon, rio, $\mathrm{y}$ villeta.

Itijan, monte.

Iualit, rio.

Iuanaan, isla.

Iuisan, punta.

Ivisan, pueblo.

Jabualla, isla.

Jabujab, punta.

Jacbag, punta.

Jaclugan, bahia.

Jaen, puelslo.

Jagdan, punta.

Jagna, putblo.

Jala, punta.

Jalaba, punta.

Jalajala, alto, casa hacienta, pueblo, y punta.

Jalaur, rio.

Jaldan, puntil.

Jalian, isla.

Jaligue, rio, y villeta.

Jaligue, or Tajaligue, punta.

Jaljat, monte.

Jalo, rio.

Jalobon, rio.

Jamelo, ensenarla, y puerto.

Jamuraon, ensenarla, islote, y purta.

Janabatas, canal.

Janabon, isla.

Janaojanao, ensenada.

Jandayan, isla.

Jangan, punta.

Janipaan, rio.

Janoyoy, isla.

Janto, isla.

Japitan, rio.

Jaro, laguna, pueblo, y rio.

Jasaan, pueblo.

Jau, isla.

Jaulo, isla.

Javiel, montes, y junta.

Jayaang, isla.

Jervois, punta.

Tesus, punta.

Jibatan, punta, rio, y villeta.
Jibitnil, isla.

Jidulpan, isla.

Jilagtungan, isla.

Jilaitin, punta.

Jilandargan, isla.

Jilantaguan, isla.

Jimalalud, pueblo.

Jimamaylan, jueblo.

Jimuguit, funta.

Jinablan, baluarte.

Jinabuyan, villeta.

Jinalnian, villaje.

Jinamoc, isla.

Jinatungan, junta.

Jinaupang, punta.

Jindan, rio.

Jingagatan, punta, rins, y seno.

Jingooc, rio, y villaje.

Jinolanan, punta.

Jintotolo, isla, (de) pawo, y punta.

Jinutangan, isli.

Jobo, islote, punta, y villaje.

Jolo, archipielago, capital, ensenarla, fondeadero, islas, y mar.

Jomalig, isla.

Juac, isla.

Juambuyod, isla.

Juanico, estrecho.

Juaya, punta.

Jubag, rio, y villeta.

Jubalan, puerto.

Juban, ensenada, y pueblo.

Jubas, punta.

Jubasan, punta.

Julugas, puellu.

Junop, punta.

Junuguit, rio.

Jurao-jurao, isla.

Juri, punta.

Kabingan, isla.

Kaladis, monte.

Kakalmund, estero.

Kalambugan, rancheris, y seno.

Kalinahan, estero.

Kalublu, isla.

Kalupag, isla.

Kalut, isla.

Kanipa, punta.

Kansina, isla.

Kapual, isla.

Katunot, rio.

Kauluan, isla.

Kaulungan, isla.

S. Doc. $228-3$ 
Keenapoussan, isla.

Kestrel, bajo.

Kudal, isla.

Kulasejang, baj», punta, y rio.

Kulassun, islas.

Kwa-liang, bahii:.

Laa, isla.

Labangan, rio.

Labijao, punta.

Labiranan, funta.

Labno, isla.

Labo, monte, pueblo, punta, y rio.

Labot, monte.

Labuan, rio.

Labugan, seno.

Labut, punta.

Labuyo, arroyo.

Lacapon, rio.

Lacay-lacay, punta.

Lactag, punta.

Lacutan, seno.

Lacy, ensenata, y pueblo.

Lagan, puntil.

Lagassan, ,ueblo.

Lagat, iska, y villeta.

Lagbangan, punta, y rio.

Lagen, isleta.

Lagnay, islote.

Lagonbato, punta.

Lagonoy, harra, pueblo, y seno.

La Granja, fueblo, y semo.

Laguan, ensenala, isla, jueblo, y semo.

Laguimanoc, bantay, y puerto.

Laguimbanua, pinta.

Laguin, estero, y seno.

Laguinit, seno.

Laguma, punta.

Lagundi, arroyo, y pueblo.

Lahat-lahat, islas.

Lahi, junta, y rio.

Lahuy, isla.

Laja, isla.

Lajan, punta.

Lajanosa, isla.

Lajas, islas.

Lajol, punta.

Lajon, punta.

Lakiktipac, isla.

Lakits, islas.

Lala, arroyo.

Lalabunguen, seno.

Lalauigan, islote, y punta.

Lalaya, rio.

Lalayan, rio.
Lalayunga, punta.

Lalay-yagan, punta.

Lal-lo, pueblo.

Lalunga, isla.

Lalutaya, isli.

Lamalana, punt:a.

Lamao, punta, y rio.

Lambajon, punta.

Lambangan, rio.

Lambillon, funt:ı.

Lambusan, punta.

Lamesajan, punta.

Lamigan, puerto, y punta.

Lamigon, punta.

Laminao, villaje.

Lamingao, isla.

Laminusa, islas.

Lamit, isla.

Lamon, balicia, y jumta.

Lampinigan, isla, y rancheria.

Lampon, puerto.

Lamud, isleta.

Lamuyon, or Solitario, isla.

Lanahuan, isla.

Lanan, rio, y visita.

Lanang, pueblo, y punta.

Lanao, or Malanao, laguna.

Lanas, monte.

Lanat, monte.

Landargun, monte.

Landican, punta.

Landungan, punta.

Landusan, seno.

Langan, rancheriı.

Langao, punta.

Langasmate, islis.

Langcan, puerto, y punta.

Langoagoan, monte.

Langos, viliaje.

Langoy, isleta.

Langtanag, punta.

Languipao, isla.

Languyon, punta.

Lanhil, islas.

Lanibga, junta, y rio.

Lanigan, puerto.

Lankayan, isla, y puerto.

Lantao, isla.

Lantian, punti.

Lantuba, punta.

Lanumang, rio.

Laoag, pueblo, y rio.

Laohauan, rio.

Laon, pueblo.

Lapa, monte. 
Lapac, isla, monte, y punta.

Laparan, islia.

Lapat, punta.

Lapinig, arroy.

Lapinin, islas.

Lapirauan, villaje.

Lapitan, punta.

Lapog, ensenada, fondeadero, y putblo.

Lapog, or Solotsolot, bahía.

Larga, punta.

Lasang, punta, y rio.

Lasisi, punta.

Lasuan, isla.

Lasy, seno, y villaje.

Latun, villaje.

Latungon, monte, y yunta.

Lauag, villaje.

Lauajan, villeta.

Lauang, pueblo, y rio.

Laugugon, punta.

Lauing, ensenada.

Lauis, pueblo, y punta.

Lauy, punta.

Lavampo, isla.

Lavezares, pueblo.

Lavigan, fondeadero.

Laya, rio.

Layaban, punta.

Laya-laya, isla.

Layapac, junta.

Laylay, rio.

Lazarino, bajo.

Lean, isleta, y monte.

Lechones, punta.

Lectuan, punta.

Ledan, monte.

Legaspi, pueblo.

Legazpi, nonte.

Lekud, villaje.

Lemery, pueblo.

Lemondo, isla.

Lenungan, islat.

Leon, islas.

Lesa, seno.

Letayen, isli.

Letue, punta.

Ley, estero.

Leyte, islas, y pueblo.

Lian, pueblo, y punta.

Lianga, ensenarla, fuerte, puel, ls, y punta.

Liao, ensenada.

Libaganon, rio.

Libagao, rio.

Libagon, villeta.
Lival, punta.

Libang, rio.

Libango, junta.

Libas, isla, pueblo putertı, punta, y rio.

Libay, pueblo, y junta.

Liberanan, punta.

Libjo, pueblo.

Libog, pueblo.

Liboho, puerto.

Libon, rio.

Libucan-Daco, isla.

Libucan Gutiay, isla.

Libutu, islas, y rio.

Licanin, rio.

Licoc, punta.

Licoco, isla.

Licohan, villaje.

Liglio, junta.

Ligpo, punta.

Liguasan, laguna.

Lijanang, punta.

Lila, pueblo, y punta.

Lili, punta.

Lilicusun, pinta.

Liloan, isla, pueblo, puerto, y punta.

Limaa, punta.

Limangangan, villeta.

Limangao, isla.

Limao, rancheria.

Limapatay, estero.

Limasaua, isla.

Limay, punta.

Limbancauayan, isla, pherto, y villeta.

Limboc, punta.

Limbones, isla, y punti.

Limbujan, rio.

Limisi, isla.

Linao, barrio, foncleatero, islat, puntal, rio, seno, y villije.

Linaon, seno.

Linapacan, islas, pueblu, y purtu.

Linda, isla.

Lindug, lio.

Linek, rancheria.

Linganay, junta.

Lingayen, caltital, y golfo.

Lingit, villeta.

Linguisan, isla.

Linoa, islute.

Lintancan, isla.

Lintayan, rio.

Lintian, punta.

Lintogut, rio.

Lintucan, esterw.

Lion, seno. 
Liong, fuerte.

Lipa, pueblo, funta, y semo.

Lipado, punta.

Lipata, monte, y junta.

Liruan, villeta.

Lisay, punti.

Litabangan, junta.

Litapan, arroyo.

Livas, isla, y punta.

Livata, harrio.

Liza, punta.

Llorente, monte.

Loay, pueblo, punta, y rio.

Loboc, puelilo.

Loboo, monte, pueblo, y punta.

Locangan, rio.

Loco-loco $u$ Sigayan, punta.

Loculan, hajos, foncleatero, pueblo, punta, y rio.

Lode, seno.

Lokdatan, villaje.

Looc, ensenala, pueblo, funta, y rio.

Loog, bahia, puel,lo, punta, rio, y seno.

Loon, foncteaclero, pueblo, y punta.

Loran, isla.

Loro, pico.

Losan, rancheria.

Los Baños, pueblo.

Los Carabaos, islas.

Los Confites, islas.

Los Curianas, islas.

Losoc, bantay.

Loxdale, bajo.

Luangat, punti.

Luauau, bantay.

Lubalan, punta.

Luban, fondealero, puerto, y punta.

Lubang, grupo ale islas, pueblo, y punta.

Lubic, islas.

Lubo, punta.

Lubog, isla.

Lubug, hantay, y seno.

Lubung, islas, y punta.

Lubutglubut, isli.

Lucatan, monte, y villaje.

Lucena, pueblı.

Lucson, punta.

Ludican, punta.

Lugatoc or fico Talagdayan, monte.

Lugbon, punta.

Luginut, isla.

Lugsen, monte.

Lugus, isla, y punta.

Luimbia, junta.
Lumaguus, rio.

Lumancapah, punta.

Lumango, punta.

Lumanig, seno.

Lumbang, pueblo, y rio.

Lumbian, isla.

Lumbucan, (de) canal, isla, y peligro.

Lumbujan, punta.

Lumintan, bajo, punta, y rio.

Lumlum, punta.

Lumucluc, rio.

Lumunum, villeta.

Lungib, punta.

Lunlut, punta.

Lunquiqui, isla.

Lupa, isla.

Lupagan, rio.

Luput, punta.

Lusaran, punta.

Lusarang, isla.

Luson, isleta.

Lusunsacatao, punta.

Luta-lita, isla.

Lutangan, punta.

Lutao, bajos.

Lutucnan, arroyo.

Luyan, villaje.

Luyucan, ensenarla or puerto.

Luzon, isla, monte, pueblo, y punta.

Maachin, pico.

Maagpit, isla.

Maalat, isla, y rio.

Maasin, pueblo, rio, y seno.

Maayon, pueblo, y rio.

Mababas, isla.

Mabac, isla.

Mabadis, isla.

Mabahuha, punta.

Mabatu, punta.

Mabatui, punta.

Mabguban, rio.

Mabien, punta.

Mabintan, monte.

Mabiao, monte, y rio.

Mabio, bantay, y punta.

Mabitac, pueblo.

Mabo, ensenada.

Maboa, punta.

Mabolog, punta.

Mabot, punta.

Mabudis, isla.

Mabugnao, punta.

Mabungan, cabo. 
Mabusao, rio.

Mac, villeta.

Macababay, punta.

Macabalang, bantay.

Macaban, puntil.

Macabasa, punta.

Macabebe, puehlo, y rio.

Macagiuna, isla.

Macaguilir, isla.

Macaguit, punta.

Macaina, isla.

Macaitot, isleta.

Macajalar, bahia.

Macajol, rancheria.

Macalaba, isla.

Macalate, punta.

Macalayo, islote.

Macaldo, bantay.

Macalino, punta.

Macambal, villaje.

Macambol, fondeatero.

Macangani, isla.

Macao, punta.

Macaonan, punta.

Macapaga, isla.

Macapdos, punta.

Macapilay, villeta.

Macar, fondeadero.

Macaribao, rio.

Macarigay, punta.

Macasandat, pico.

Macasandor, rio.

Macati, isla, y villaje.

Macato, pueblo.

Macatul, punta.

Macaturin, volean aetivo.

Macaulit, rio.

Mac Crohon, juebls.

Machesi, isla.

Maclanas, punta.

Maclayao, monte.

Macolod, monte.

Macoto, or Canmanhali, ensenadat, y punta.

Mactan, isla.

Maction, islote.

Macugil, punta.

Maculabo, isla.

Maculagu, punta.

Maculay, isla.

Maculmacul, punta.

Macumpan, junta.

Madalog, punta.

Madang, monte, y punta.
Madaon, monte.

Madaum, fondeadero, y rio.

Madia-as, monte.

Madilao, punta.

Madrepora, punta.

Madrigal, villaje.

Madrilejo, villeta.

Madumug, isla.

Maemany, pumta.

Maestre de Campo, isla.

Magaan, villeta.

Magabao, pulı.

Magabo, punta.

Magabun, punti.

Magaguilan-guilan, islote.

Magalang, pueblo, y punta.

Magallanes, bahia, banco, ensenarla, 1)ueblo, y punta.

Magalo, punta.

Magapu, punta.

Magasauangtubig, rio.

Magaso, monte.

Magat, rio.

Magatacan, junta.

Magatalijan, isla.

Magauhan, punta, y rio.

Magay, puita.

Magayao, rio.

Magban, isliı.

Magbayan, punta.

Magcanauay, arroyo, y villaje.

Magdalena, puelilo, puerto. y punta.

Magdangai, punta.

Magdoc, monte.

Magearagui, islit.

Maggano, isla.

Maginasu, punta.

Maginoc, villaje.

Maglaban, punta.

Maglabit, punta.

Maglababon, jumta.

Maglan. baluarte. y preblo.

Maglolob, villaje.

Maglubun, punta, y rancheria.

Magoisi, isla.

Magpeos; isla.

Magsajon, rio, y villeta.

Magsalim, punta.

Magtimua, pierlra.

Magtulinog, punt:t.

Maguao, rio.

Maguican, junta.

Maguigtig, funta.

Maguinalem, monte. 
Magun, villaje.

Mahaba, isla.

Mahanay, isla.

Mahanglad, riı.

Mahinog, pueblu.

Mahuja, monte.

Maibo, rio.

Maiboc, rio.

Maibun, ensenada, y puelsh.

Maiduun, islas.

Maihagonas, riı.

Mailaban, ensendula.

Mailabu, rio.

Mainaga, ensenarla.

Mainit, pueblo.

Mainit, or Saponga, layo.

Mainonon, punta.

Maipat, (rle) canal.

Maitiaguit, isla, y villeta.

Maiyapay, monte.

Majaba, isla.

Majabibujabin, punta.

Majacob, isla, y villeta.

Majanay, isla.

Majulat, punta.

Malababon, punta.

Malabaga, rio.

Malabahoc, nonte.

Malabang, punta.

Malaboyoc, villaje.

Malabrigo, fondeadero, y finta.

Malabuctun, isla.

Malabug, seno.

Malabuyoc, pueblo.

Malacaba, isla.

Malacabang, villaje.

Malaga, rio.

Malagabon, punta.

Malagan, junta.

Malagit, villeta.

Malagui, pulo.

Malaguinoan, isla.

Malagundi, $o$ Galban, junta.

Malagusan, punta.

Malagutay, rio.

Malahuit, rio.

Malajibomanoc, isla.

Malajob, rio, y villeta.

Malajon, isla.

Malalag, perto, rio, y sems.

Malalimon, punta.

Malalod, monte.

Malamaui, isla, y juerto.

Malamis, isla.
Malampaya, lahia, puerto, y rio.

Malanao, isla, punta, y rin.

Malanao, or Lanao, layo.

Malanay, pueblo, y punta.

Malancauas, nonte.

Malandoc, rio.

Malangaban, isla.

Malanipa, isla.

Malanto, pueblo, y junta.

Malanut, comandaneia, nonte, sierra, y villeta.

Malaoton, isla.

Malapackum, isla.

Malapascua, (de) canal, y iwla.

Malapascua or Luzon, i.wa.

Malapina, isleta.

Malaposo, isletus.

Malarad, isla.

Malarat, isla.

Malaspina, or Canlaon, voluan artivo.

Malatag, punta.

Malatandan, punta.

Malatanguit, junta.

Malate, pueblo.

Malatpuso, isletid.

Malatumbaga, punta.

Malatuna, punta.

Malaubang, punta, y rio.

Malay, pueblo.

Malaya, corlillera, isla, y villeta.

Malaylay, rio.

Malayo, punta.

Malayoc, punta.

Malayog, punta.

Malazimbo, isla.

Malbatan, isleta.

Malbato, rio, y villaje.

Malbinchilao, isleta.

Malburan, rio.

Malcampo, seno, y villeta.

Malcapuyo, isleta.

Malcatop, isleta.

Malebol, rio.

Malecon, pueblo, y junts.

Maleput, rio.

Malesbugui, punta.

Malhon, isla.

Maliao, monte, y rio.

Malibago, puetslo, y punta.

Malicaboc, isla.

Malicabog, islit.

Malicay, ensenarla, y seno.

Malicut, isla.

Mralifut, rio. 
Maligay, punta, y seno.

Maligboc, puej,lo.

Maligui, rio.

Malilipot, puehlo.

Malimus, punta.

Malinao, or Buhi, monte, puss, puert", y rio.

Malindang, monte.

Malingui, isla.

Malipano, fondeadero, is il, y punta.

Malipu, hahia, y punta.

Malita, punta.

Malitbog, monte, pueblo, y rio.

Malitinao, rio, y villeta.

Maliuga, isla.

Malnijat, isleta.

Malobog, punta, y rio.

Malocot, punta.

Malolo, monte.

Malolos, neblo.

Malootone, isla.

Maloro, arroyo, y seno.

Malpacao, isleta.

Maltatayoc, isleta.

Maitolpoc, isleta.

Maluan, rio.

Malubaroc, punta.

Malubug, seno.

Malubutglubut, i.la.

Maluc, fondearlero.

Malugan, villaje.

Malumahuan, isla.

Malumbacong, arroyo, y funta.

Malupali, rio.

Malusacusac, punta.

Maluso, bahia, pueblo, y rio.

Maluson, rio.

Maluya, punta.

Mamad, isla.

Mamailan, junta.

Mamali, rio.

Mamaluy, rio.

Mamanao, punta.

Mamanga, rio.

Mamanoc, isla.

Mamanuk, islas.

Mambagi, hajo, y pueblo.

Mambajab, punta.

Mambajao, pueblo. .

Mambajun, punta, y rio.

Mambulao, pueblo, puerto, y puntat.

Mamburao, bajo, pueblo, y rio.

Mamebel, rio.

Mampagut, easerios.
IMamucu, rancheria.

Mamvanon, villeta.

Manaba, rio.

Manacnac, punta, y seno.

Manadi, isla.

Managaysay, punta.

Managuion, hahia.

Manalumo, punta.

Manamoc, islas.

Mananga, rio.

Manangal, isla.

Manangulä, puntiı.

Manantial, villaje.

Manapla, meblo, y rio.

Manat, rio.

Manay, pueblo, y rio.

Manbajao, villaje.

Mancahuron, isla.

Mancaris, punta, y villeta.

Mancayangui, isla.

Mandalagan, monte.

Mandalugan, rio.

Mandan, isla.

Mandaon, puerto, punta, y villeta.

Mandauan, fordeadero.

Mandaue, pueslo.

Mandaui, iwlas, y rio.

Mandavy, Jaguna.

Manday, estero.

Mandonga, punta.

Mandug, rio.

Mangal, puntix.

Mangarin, pueblo, puerto, y lunta.

Mangaro, punta.

Mangas, punta.

Mangayao, punta.

Mangcao, punta.

Mangenguey, isleta.

Mangla, punta.

Mangle Butuan, islia.

Mangobobe, isla.

Mangrove, punta.

Mangsee, (de) canal.

Mangsee del Sur, isla.

Manguina, ric.

Mangurucuru, villaje.

Manhal, rio.

Mani, monte.

Manicaa, rio.

Manicaan, visita.

Manicari, fondeadero, y isla.

Manicolat, islas.

Manigil, isla.

Manigonigo, isla. 
Maniguin, isla.

Manila, (de) bahia, capital, y puerto.

Maniloc, isla.

Manimbulao, isleta.

Maninagan, isla.

Maninila, rio.

Manipul, rancheria.

Manipulon, isla.

Manisbasco, punta.

Maniuayan, isleta.

Manjao, rio.

Manjud, punta.

Manjuyoc, punta, y villeta.

Manjuyod, pueblo, y punta.

Manlagule, isla.

Manlanat, isla.

Manliog-liog, punta.

Manoc, isla, y punta.

Manoc-manoc, islas.

Manog, punta.

Manoyaosayao, punta.

MIanraya, villaje.

Mansalay, ensenarla, y pueblo.

Mansanga, rio.

Mansiol, ensenadi, pmello, y punta.

Mansulung, rio.

Mantacao, isla.

Mantabuan, isla.

Mantag, pueblo, y punta.

Mantaid, isla.

Mantalinga, irla.

Mantalingajan, monte.

Mantangule, isla.

Mantaquin, bahia.

Mantay, isla, y punta.

Mantibao, punta.

Mantitinao, rio.

Mantoconan, isla.

Mantuaca, punta.

Mantulary, isleta.

Manubo, isla.

Manuc-IManca, isla.

Manud, isla.

Manumitan, barrio.

Manungut, rio.

Manurigao, villeta.

Maotonon, isleta.

Mapanga, fondealero, y seno.

Mapatag, punta.

Mapiangan, lieo.

Mapilio, punta.

Mapinjor, punta.

Mapotao, isła.
Mapuapantan, punta.

Mapusa, villeta.

Maputi, punta.

Maputian, or Arenas, punta.

Mapuyo, punta.

Maqueda, bahia, y (de) canal.

Maquilao, arroyo, y punta.

Maquiling, volcan apagarlo.

Maquiliquian, punta.

Maquiliquil, punta.

Maquinag, punta, y villeta.

Maquinit, isletas.

Marabay, cordillera, y punta.

Marabu, bajo.

Maracañao, islas.

Maradionan, villeta.

Maragat, villeta.

Maralison, isla.

Marama, rio.

Maranding, rio.

Marangas, villaje.

Marangay, punta.

Marantao, isla.

Marantow, punta.

Marapatang, fondearlero.

Marara, ensenada.

Marasi, lahia.

IIarayo, villaje.

Marban, hinco.

Marcalon, pueblo.

Mardagao, rio.

Marga, seno.

IMargajo, jinta.

Maria, islote.

Maria Antonia, puerto.

IIaria Cristina, puel,lo, y seno.

Marianas, punta, y rio.

Maribojoc, ensenada, y pueblu.

Maricaban, isla, (de) pasis, y villaje.

Maricaban, (de) pasos N. y.

Marigabato, punta.

Marigatdan, punta.

Marigil, punta.

Marigondon, punta, rin, y villeta.

Marihatas, villaje.

Marily, isla.

Marinap, barrio, punta, y senu.

Marinduque, isla.

Marinon, isla.

Irarintoc, punter, y rio.

Maripipi, isla, y pueblo.

Mariqui, punta, y rio.

Mariquit, isla, y punta.

Mariquitdaquit, islote. 
Maririg, isla.

Mariveles, monte, puehlo, puerto, y punta.

Marlanga, or Tablazo, ensenada, monte, pueblo, y punta.

Marongas, islas.

Martin, rerro.

Martine, punta.

Marurugao, rio.

Masaguisi, rio.

Masahuron, isla.

Masala, punta.

Masapilit, punta

Masaya, rio.

Masayoc, rio.

Masbate, isla, y pueblo.

Masi, pueblo, y rio.

Masin, isla, y rio.

Masingal, seno, y villaje.

Masingil, isla.

Masinloc, ensenada, fomleaclero, monte, pueblo, prerto, y rio.

Masisi, villaje.

Masisingi, isla.

Maslic, punta.

Masulag, isla.

Mataas Pang-pang, punta.

Matabao, isla.

Matablan, rio.

Matabul, rio.

Matacon, ensenarla, y pueblo.

Matagda, isla.

Mataja, isla.

Matalang, rio.

Matalan-talan, punta.

Matalom, juehlo.

Matalvi, puerto.

Matampoy, arroyos, y punta.

Matanal, monte.

Matanat, isla.

Matanaye, isla.

Matancan, ensenada.

Matandumaten, isla.

Matangal, inonte, y punta.

Matanguingue, villaje.

Matanpay, estero,

Matarabis, islas.

Matarinao, punta.

Matatindog, jumta, y rio.

Mataya, isla.

Matayon, punta.

Mateo, pueblo, y punta.

Mati, comandancia, fondearlero, rio, y villeta.
Matiao, ensenada, fondealero, y rin.

Matigboc, rin.

Matilay, rancheria.

Matimus, seno.

Matinloc, isla y jermun.

Mationg, villeta.

Matitinao, ensenarla.

Matnog, ensinarla, pueblo, rio, y ч'mo.

Matocot, punta.

Matos, banco.

Matubug, ensenada.

Matulimog, punti.

Matulin, isla.

Matumut, rio.

Matung, punta.

Matuntun, monte, y pinta.

Matutinao, pueblo.

Matutindog, punta.

Mauban, jueblo.

Mauis, isla.

Maulana, seno.

Maulbaal, villaje.

Maulingon, punta, y seno.

Maulun, punta.

Maumaun, isla.

Mauo, rio, y villeta.

Maupan, punta.

Maya, rio.

Mayabacan, isleta.

Mayanga, isla.

Mayan-Payan, isleta.

Mayao, villeta.

Mayjan, villaje.

Mayllague, punta.

Mayluban, rio.

Mayo, sello.

Mayon, rolcan activo.

Mayondol, monte.

Mayraira, punta.

Mayuga, junta.

Meara, isla.

Melchor, islas.

Mellan, or Calumpan, rio.

Melville, abus.

Menar, islote.

Meol-meol, punta.

Mercedes, pueblo.

Mereaga, rio.

Merida, pueblo.

Mesa, isla, monte, y (de la) junta.

Miaga, rio.

Miagao, pueblo, y punta.

Migcaniguin, muntil. 
Migpanguit, or Panguil, rio.

Milagbiga, ensenatla.

Mimiloc, isleta.

Minadion, islote.

Minaga, purta, y rio.

Minaloa, islote.

Minalolo, isla.

Minalos, punta.

Minalutan, junta.

Minaman, isla.

Minangas, puerto, y junta.

Minanganalandan, villaje.

Minanut, isla.

Minapan, monte.

Minasacan, rancheria.

Minasangan, isla.

Minasaua, isla.

Minatulan, seno.

Mindanao, isla, y rio.

Mindoro, (de) estrecho, y isla.

Minglanilla, pueblo.

Mingolit, monte, y rio.

Minic, fomleadero.

Mininlay, isleta.

Minis, islas.

Minolo, fonclealero.

Mintag, punta.

Minuluan, pueblo.

Minusuang, rio.

Mira, villaje.

Miranda, villaje.

Mirigil, punta.

Misamis, distrito, foncleatero, puthlo, y junta.

Misanga, islas.

Misibis, punta.

Misua, ensenacla.

Mitra, monte.

Miunel, punta.

Miut, villeta.

Mobanen, isleta.

Mobary, islas.

Mobo, ensenarla, pueblı, rio, y seno.

Mocaboc, punta.

Moises, monte.

Molane, isla.

Molboc, punta.

Mompog, isla, y (le) paso.

Moncada, tneeilarla, y jneblo.

Mondeo, rio.

Mongalao, islas.

Mongos-mongos, isla.

Monja (La), isla.

Monserrat, monte, y puetilo.
Monte, (del) punta.

Montufar, pueblo, y punta.

Monuangon, arroyo.

Moranon, rio.

Morigaya, rio.

Morigue, isla.

Moroc, rio.

Moron, pueblo.

Moroporo, isla.

Morsoom, cato.

Moug, rio.

Mual, seno.

Mualbaal, villaje.

Mucabog, isla, y punta.

Muda, islas.

Muelle, punta.

Mulanay, fondealero, y pueblo.

Muleegee, islas.

Mulita, rio.

Munti, pulo.

Muntinglupa, pueblo.

Muquid, rio.

Murcielagos, hahia, y islotes.

Mursoom, punta.

Musa, puehlo, y puerto.

Musasole, juehlo.

Naasin, pueblo.

Nabagbagan, punta.

Nabalas, villeta.

Nabanoc, punta.

Nabao, rio chico, y rio grande.

Nabasan, punta.

Nabat, isleta.

Nabbuangan, rio.

Nabirinaya, punta.

Nabitas, rio.

Nabotas, villaje.

Nabucuban, nunte.

Nabugat, islas.

Nabugtu, isla.

Nabugtut, isla.

Nabulao, ensenada.

Nabuluan, rio.

Naburut, isla.

Naburaoburao, isla.

Naburos, isla, y monte.

Naburut, isla.

Nabutas, punta.

Nacaban, punta.

Nacopot, punta.

Nadulao, islia.

Naga, arroyo, pierlras, puehlo, punta, y rio.

Nagaba, pueblo. 
Nagapu, monte.

Nagarao, isla.

Nagas, puehln, y punta.

Nagbuclasan, junta.

Naglabo, rio.

Naglagabon, seno.

Naglanguay, punta.

Naglon, puntal.

Naglumbang, punta.

Nagmagpacan, villaje.

Nagpalit, , inta.

Nagpartian, jueblo.

Nagtig, isla, y punta.

Naguban, isla.

Nagubat, islas.

Naguiba, punta.

Naguionca, printa.

Nagumbuayan, punta.

Nahuan, isla.

Naig, pueblo, punta, y rio.

Nailug, (m Silla Hendida, nonte.

Nailog, punta, y rio.

Nailon, monte, y punta.

Naindain, punta, y rio.

Naisog, punta.

Naisot, ensenada, y villeta.

Najibil, punta.

Nakoda, isla.

Nalaut, isleta.

Nalibos, isla.

Nalibunan, rio.

Nalunga, isla.

Nalupa Nuevo, pueblo.

Namagpacan, ensenada, y puelilo.

Namanuco, junta.

Namatayan, ris.

Namatian, isla.

Namulugpulug, punta.

Nanca, pueblo, y punta.

Nanga, islas, y punta.

Nangalao, islas.

Nangca, seno.

Nangi, village.

Nanguan, isla.

Nanoc, punta.

Nan-pulo, punta.

Nan-sha, cabo.

Nanu, isla.

Nanuntugan, isla.

Napacao, pueblo, y punta.

Napalungan, punta.

Naparican, funta.

Napatong, punta.

Napayauan, isla, y villeta.
Napayon, islute.

Napo, punta, y rio.

Napula, isleta, y junta.

Naquebradajan, punta.

Nara, punta.

Naranjos (Los), gripu de islas.

Narbacan, villaje.

Nariz, (de la) punta.

Naro, pueblo, y senu.

Naro Chico, isli.

Naro Grande, isla.

Narro, isla.

Narvaez, haju, y banco.

Nasagui, rio, y villagee.

Nasbutta, arrerife, canal, y isla

Nasipan, punta.

Nasipit, puelılo, y puerto.

Naso, punta.

Nasog, punta.

Nasoque, punta.

Nassiping, villaje.

Nasubata, islat.

Nasuduan, monte.

Nasugbu, ensenada, fondeadero, puel,o,

y punta.

Natimbunan, isla, y villeta.

Natungtugan, isla.

Nauai, isla.

Nauco, punta, y villaje.

Naufragio, caloo.

Naujan, fcnilearlero, laguna, pueblo, punta, y rio.

Naulo, punta.

Navahay, islote.

Naval, pueblo.

Navitas, punta, y villeta.

Navotas, pueblo, y rio.

Naya, punta.

Nayun, fondearlero, y rio.

Nazasa, fondearler, y seno.

Negra, punta.

Negron, monte.

Negros, isla.

Neiba, rio.

Niaporay, isleta.

Niarro, isla.

Nibobon, rio.

Nin, bahia, y bajos.

Ninamon, rio.

Nipa, baluarte, punta, y rio.

Nipa-nipa, estero, punta, seno, y villeta.

Niugan, rio.

Noche Buena, (de) ('anal.

Nogas, isleta. 
Nonol, pueblo, y punta.

Nora, punta.

Noreste, punta.

Norte $o r$ Colorada, punta.

Norte de Balabac, estrecho.

Notable (Pico), monte.

Nucas, isla.

Nuin, fondeadero, y villaje.

Nulingin, estero.

Nuluncan, punta.

Numancia, villeta.

Nunez de Prado, bajo.

Nungay, punta,

Nunu, isla.

Nunun, punta.

Oacan, $m$ Binay, punta.

Oanio-an, isla.

Obando, pueblo.

Obelisco, villaje.

Obispo, estero.

Obon, punta.

Obong, rio.

Ocho, monte.

Ochoa, bajo.

Oco, islas.

Ocre, punta.

Octoc, punta.

Odel, isla.

Odiong, pueblo, y unnta.

Odiongon, ensenarla, y fomlearler.

Odiungan, puelilo.

Odoc, punta.

Oeste, (del) prunta.

Oeste de Basibuli, (del) canal.

Oeste de Banaran, (del) canal.

Ohitaya, isla.

Okayan, funtil.

Olagipan, pulı.

Olango, isla, y pi(w).

Olo, rio.

Olongapo, puerto.

Olutanga, isla.

Olutaya, isla.

Omon, punta.

Onas, jsla.

Ontgol, pint:l.

Oot, punta.

Opac, punta.

Opol, fondeadearo, pueblo, y junta.

Opon, puelslo.

Opong, rio.

Orani, jueblo, y rio.

Oras, ensenada, lunta, rio, y villaje.
Orayon, barrio.

Ordonez, bajo.

Orejas de Liebre, grupn de islas.

Orell, isla.

Origon, junta.

Orion, monte, y puelslo.

Ormoc, bahia, pueblı, y puerto.

Oroquieta, villaje.

Oslob, punta, y villaje.

Ostras, (de las) ensenada, y punta.

Otalora, monte.

Oton, banco, y pueblo.

Oyaquitan, villaje.

Oyon, punta.

Pabellones, isleta.

Pablo, bahia.

Pacahan, or Habang, punta.

Pacduhanan, monte.

Pacijan, isla.

Paco, pueblo.

Padada, punta.

Padang, punta.

Padidu, or Pulongui, estero.

Padlahan, rio.

Padnanungan, junta.

Padulog, punta.

Paesan, monte.

Paet, punta.

Paete, pueblo.

Pagagpac, punta.

Pagalac, juelylo.

Pagalamatan, estero.

Pagalungan, punta, y rancheria.

Pagapas, or San Pedriño, ensenarla.

Pagarian, ensenada.

Pagatpat, punta.

Pagauanen, punta.

Pagbabangunan, rio.

Pagbajan, punta, y rio.

Pagbilao, ensenarla, puelılo, rio, y'seno.

Pagbilao Chico, isla.

Pagbilao Grande, isla.

Pagbulungan, monte, y punta.

Pagdanam, bahia, cordillera, y punta.

Paghuliran, seno.

Paglalasugan, punta.

Paglicangan, [)unta.

Paglugaban, isleta.

Pagquiputan, punta.

Pagsan, monte, y rio.

Pagsanguin, villaje.

*Pagsanhan, pueblo, puerto, punta, y rio.

* The Coast Survey atlas has Pagsanhan, rio (map 18), Pagsanhan, punta (p. 19) and Pagsanjan, pueblo, y puerto (p.11.). 
Pagua, rio.

Paguirira, punta.

Pahat, rio.

Pahuat, rio.

Paiuan, villaje.

Paklahatan, villaje.

Paknam, hajo.

Pala, islas.

Palad, bajo.

Palag, ensenarla.

Palaguan, puntit.

Palajangan, isla.

Palalagoya, punta.

Palalaguejat, punta.

Palamata, rancheria.

Palana, punta.

Palanan, ensenada, y pueblo.

Palanas, monte, pueblo, y rio.

Palanguitin, punta.

Palanit, punta, rio, y villeta.

Palanjagan, isla.

Palanog, pueblo, y puerto.

Palanquitas, punta.

Palaopao, monte.

Palapa, canal, monte, puerto, y villaje.

Palapag, pueblo, puerto, y punta.

Palasan, islas.

Palaspas, punta.

Palatuan, punta.

Palaui, isla, y rio.

Palauig, arrecifes, ensenada, pueblo, y punta.

Palcocotan, isleta.

Palijon, isla.

Palina, punta, y rio.

Palipinaluajan, punta.

Palita, isla.

Paliton, punta.

Paliuan, punta.

Palixet, rio.

Palliagan, isla.

Palmabrava, isla.

Palo, pueblo, (de) punta, y rio.

Palompon, cerro, pueblo, puerto, y punta.

Palonan, villaje.

Palos, monte, pueblo, y rio.

Paloyac, islas.

Paluan, bahia, ensenada, y pueblo.

Palubu, rio.

Palumbanes, islas.

Palupari, isleta.

Paly, islas.

Pamagsalan, rio.
Pamagunan, estero.

Pamalican, isleta.

Pamang, estero.

Pamanpangon, punta.

Pambujan, bahia, pueblu, puerto, y rio.

Pamian, or Estancia, rio.

Pamilacan, isla.

Pamintangan, punta.

Paminuitan, pueblo.

Pamitinan, islas.

Pamoboan, puntil.

Pamocan, punta.

Pamontalon, isla.

Pampan, pueblo.

Pampandugang, punta.

Pampanga, seno, y villaje.

Pampat, punta.

Pamplona, bahia, pueblo, y rio.

Pamuntangan, bajo, y punta.

Panabilan, villeta.

Panabuan, estero.

Panabulon, isla.

Panabutan, ensenada, punta, y rio.

Panacan, ensenada, y rio.

Panadlihan, punta.

Panaga, monte.

Panagatan, islas.

Panagocdoc, rio.

Panagsagon, punta.

Panahonga, punta.

Panain, punta.

Panalangan, punta.

Panaliguan, rio.

Panalipan, punta.

Panalisa, punta.

Panalsalan, arroyo.

Panaluran, ensenarla, y puerto.

Panampalan, punta.

Pananamitan, punta.

Panandagan, rio.

Panang, esteru.

Panaon, (de) estrecho, isla, y funta.

Panaran, colina.

Panatiguan, rio.

Panaun, punta.

Panay, islas, pueblo, punta, y rio.

Pancol, villaje.

Pandaguitan, rancheria.

Pandalusan, isla.

Pandami, isla, y pueblo.

Pandan, ensenada, islas, monte, pueblo,

punta, rio, y villeta.

Pandanan, isla.

Pandanang, punta. 
Pandano, isla.

Pandanou, isla.

Pandanpandang, pueblo.

Pandarochan, bahia.

Pandasan, fomrlearlerr, y isla.

Pan de Azucar, isla.

Panducan, islas, y punta.

Pandugas, isla.

Pandulungan, punta.

Panga, punta.

Pangabuan, arroyo.

Pangalancalangan, isla.

Pangaldauan, isleta.

Pangaliban, punta, y rio.

Panganaa, isla.

Panganac, isla.

Pangana Paluruan, isla.

Panganiran, or Cadburanan, punta.

Panganuan, grupo de islas.

Panganuran, punta.

Pangao, punta.

Pangapasan, isla.

Pangasahan, canal, y punta.

Pangasinan, isli.

Pangatatan, isleta.

Pangdan, isla, y monte.

Pangi, senu.

Panglao, ensenala, isla, y pueblo.

Pangpang, junta.

Pangpangon, rio.

Pangtail, punta.

Pangubatan, fondeaderı, y junta.

Panguian, punta.

Panguil, bahia, lago, y puel,b.

Panguilinan, bajus.

Panguiputan, punta.

Pangulasian, isleta.

Pangusan, punta.

Pangutaran, islas, y (de) pa:o.

Panhapuyan, isla.

Paniguran, punta.

Panilahan, rio.

Panimusan, punta.

Paninihian, punta.

Panique, punta.

Paniqui, isla, y pueblo.

Paniquian, isla.

Panirugan, punta.

Panjan, punta.

Panjumajan, grupo de islas.

Panno, punta.

Pannuyan, punta.

Panpan, juntit.

Panpang, punta.
Pansiguican, pueblo.

Pansina, villaje.

Pansipit, rio.

Panta, visita.

Pantalan, punta.

Pantao, fondeadero.

Pantocomi, punta.

Pantocunan, islas.

Panubijan, islas, y uronte.

Panubulon, isla.

Panugutan, rio.

Panuitan, islas.

Panusuhan, isla.

Paoay, lago, y pueblo.

Paombo, villaje.

Papachin, isleta.

Papagdayan, rio.

Papahag, islas.

Papanayang, punta.

Paquia, isla.

Paquit, villaje.

Paquit-Paquit, rio.

Paracale, ensenada, pueblo, y punta.

Paragua, (de la) canal.

Paran, punta.

Paranan, ensenada, y villaje.

Parañaque, pueblo.

Paranas, pueblo.

Parang, bahia, islote, y pueblo.

Parangan, isla.

Paranjo, punta.

Paran-paran, comantamcia, y villaje.

Parara, punta.

Parasan, isla.

Paray-Paray, rio.

Parian Kisap, pueblo.

Paric, or Dolores, monte, y pueblo.

Parmidiaran, punta.

Paropandan, punta.

Parpagoja, pueblo.

Parudo, rio.

Pasacao, (de) ensenada, fondeadero, y pueblo.

Pasahan, villaje.

Pasal, punta.

Pasanjan, punta.

Pasay, villaje.

Pasco, (de) canal, y punta.

Pascual, puerto.

Pasegan, isla.

Pasegan Guimba, isla.

Pasencoan, isla.

Pasig, isla, pueblo, y rio. 
Pasigajon, punta.

Pasig-Pasilan, isla.

Pasijan, isla.

Pasil, bajo, y punta.

Pasit, punta.

Pastora, punta.

Pasumacanan, rio.

Pasuquin, pueblo.

Pata, fondeadero, islas, y punta.

Patanaon, isla.

Patapat, monte.

Patayan, isleta.

Pateros, neblo.

Patian, islas.

Paticolo, islas, y pueblo.

Patnanongan, islas.

Paton, isla.

Patoyac, islas.

Patuco, fondeadero, y puerto.

Patung, villaje.

Patunga, islas.

Patungan, ensenada.

Paturuan, isla.

Patyacan, punta.

Pauay, punta.

Pauican, punta, rio, y villeta.

Pauisan, punta, y villeta.

Pauros, monte.

Payabon, villaje.

Payo, islas, y pueblo.

Payopot, barrio.

Paypay, or Lambajan, punta, y villeta.

Paytudland, punta.

Paz, isla, y pueblo.

Pedregosa, isleta.

Pedrino, monte, y punta.

Pedruscos, punta.

Peligro, monte.

Pelote, punta.

Peninsula, punta.

Peñascales, punta.

Peñascosa, punta.

Peñon de Coron, islas, y pueblo.

Pepitas, isla.

Pequena, isla.

Peris, envenada.

Perseus, bajo.

Pescado, punta.

Pescador, (del) isla.

Peters, cabo.

Petisa, punta.

Pezon, monte.

Piacan, punta.

Piagao, punta.
Pialun, rio.

Piapi, punta.

Pico de Loro, monte.

Picol, punta.

Picos Tagun, villaje.

Picuda, isla, y punta.

Picudo, monte.

Piedras, (de) punta.

Pig, punta.

Pigcarangan, arroyo.

Pignadagatan, punta.

Pila, pueblo.

Pilar, isla, pueblo, y pierto.

Pilas, (de) canal, y islas.

Pili, monte, y pueblo.

Pililla, pueblo.

Pilis, enrenada.

Pilisan, punta.

Piña, isla, y seno.

Pinacanauan de Cabagan, rio.

Pinacanauan de Tuguegarao, rio.

Pinacanauan de Tumauini, rio.

Pinacapan, punta.

Pinachinguyan, isla.

Pinachiuyan, isleta.

Pinacuapan, isla, y punta.

Pinaculan, punta.

Pinagbucan, punta.

Pinagcurusan, ensenada, y punta.

Pinaghaya, punta.

Pinagmupuan, punta.

Pinagnapan, isla.

Pinalabuan, punta.

Pinalit, seno.

Pinalobo, monte.

Pinamalayan, ensenaria, pueblo, punta, y rio.

Pinamitan, punta.

Pinamucan, monte, punta, y riı.

Pinamungajan, pueblo, punta, y seno.

Pinamuntangan, ensenada, y punta.

Pinandungan, punta.

Pinangomajan, villaje.

Pinas, isleta.

Pinatayan, bajo.

Pinauasan, punta.

Pinauran, punta.

Pinavisagan, ensenada.

Pineda, pueblo.

Pingano, monte, y punta.

Pinget, isla.

Pinitan, punta.

Pinos, punta.

Pintad, isla. 
Pinulacan, punta.

Pinutan, punta.

Pirara, punta.

Pirata, (del) bahia, y isla.

Piratan, rio.

Pisac Pisac, villaje.

Pitas, isla, monte, y punta.

Pitogo, fondealero, isla, monte, (de) paso, pueblo, rio, y seno.

Placer, pueblo.

Planique, punta.

Plus, isla.

Po, estero.

Pobre, isla.

Pola, jueblo, y punta.

Polac, ensenada, y jueblo.

Polauit, punta.

Polillo, isla, pueblo, y puerto.

Polloc, fondeadero, pueblo, puerto, y punta.

Polo, isla, lago, pueblo, y punta.

Polopantao, punta.

Polot, rio.

Pomelikan, isla.

Ponclian, punta.

Pongdol, punta, y seno.

Ponson, islas.

Pont, punta.

Pontado, punta.

Ponte, bajo.

Pontevedra, pueblo, y rio.

Ponti, punta.

Pontod, punta.

Pontud, bajos.

Popolcan, isleta.

Popototan, isleta.

Pora, punta.

Poro, isla, y pueblo.

Poro de los Camotes, grupo de islar.

Porong, isleta, y punta.

Portuguesa, punta.

Poruquen, barrio.

Posdey, monte.

Posedol, villaje.

Potique, punta.

Potol, punta, y rio.

Poupit, monte.

Prahu, punta.

Prat, punta.

Principe Alfonso, (del) puerto.

Promontorio, punta.

Providencia, punta.

Prueba, bajo.
Puan, isla.

Pucdul, rio.

Pucio, punta.

Pucul, estero.

Puerco, isleta.

Puerto, (del) punta.

* Puerto Princesa, capital.

Puga, grupo de islas.

Pugal, rio.

Pugao, bantay.

Pugatin, monte.

Pugguiauan, punta.

Pugpucanan, islote.

Pujada, bahia.

Puju, lajo.

Pulagay, punta.

Pulaluaac, punta.

Pulanauta, punta.

Pulanglupa, punta.

Pulangui, pueblo, y rio.

Pularaquin, villeta.

Pulipo, pulo.

Pulo, punta.

Pulo Caballo, isla.

Pulumbata, punta.

Pulupantao, isla.

Pulute, sierra.

Punas, or Loboo, punta.

Puntoc, punta.

Puntod, restinga.

Punuan, isla.

Punubulu, islote.

Punul, isla.

Punungan, isla, y pueblo.

Pusan, punta.

Pusgo, puerto, y punta.

Pusilao, rio.

Putad, rio.

Purainga, isla.

Putiao, puerto, y punta.

Putic, isleta, y punta.

Putili, islote.

Puto, punta.

Putun, pneblo.

Puya, islas.

Quebrada, (de la) monte.

Quebradas, punta.

Quebrado, calıo.

Quiadoc, islote.

Quibigahan, rio.

Quico, bajo.

Quidabun, grupo ie islas. 
Quidamak, s(’no).

Quidancac, senu.

Quidapil, punta.

Quigaanon, punta, y villeta.

Quijada, [mnta.

Quilbait, pueblo, y punta.

Quilubedan, monte.

Quimaya, ris.

Quimbaludan, isleta.

Quimbuluan, isleta.

Quiminalin, isletas.

Quimitad, villaje.

Quinabacbagan, bajo.

Quinabaranon, punta.

Quinablayan, punta, y villaje.

Quinabucasan, junta.

Quinabugan, isla.

Quinabutasan, paso.

Quinaguitnan, barrio, y rio.

Quinalang, fondeadero.

Quinalapan, punta.

Quinalasag, isla.

Quinali, punta, y villeta.

Quinaligan, isla.

Quinamaligan, isla.

Quinamanuca, isla.

Quinanad, pinta.

Quinangalang, villaje.

Quinangcang, seno.

Quinapundan, pueblo.

Quinapuntan, rio.

Quinasuyan, rio.

Quinay, villeta.

Quindabyz, isla.

Quiniluban, isla.

Quinituay, islote.

Quinuguitan, pueblo.

Quioboc, punta.

Quiot, pueblo.

Quipit, monte, y junta.

Quisao, pulo.

Quitahis, punta.

Rabin, punta.

Rabon, rio.

Ragalumbi, isla.

Ragay, ensenaria, pueblo, y seno.

Ragutaya, punta.

Rajamuda, estero.

Ramesamey, isla.

Ramillete, isla.

Ramos, isla.

Ranacan, punta.

Ranis, punta.
Rapu-rapu, isla, y (de) jaso.

Rasa, islas, y punta.

Rasa, or Majaba, isla.

Rasa, or Mantaquin, isla.

Raso, islote.

Raton, banco.

Ravis, punta.

Rawnsley, punti.

Ray, banco.

Real, punta.

Recomo, punta.

Redondo, monte.

Refugio, (del) isla.

Regatta, isla.

Reinard, isla.

Reinas, (de las) Jahia.

Remedios, villaje.

Remintao, punta.

Remus, bajo.

Reposo, punta.

Restinga, punta.

Reynoso, arrecife.

Rio Grande de Cagayan, rio.

Rio Grande de la Pampanga, rio.

Rio Grande de Mindanao, or Pulangui, rio.

Rio Grande de San Mateo, rio.

Riposet, monte.

Rita, islas.

Rivera, punta.

Rivonac, punta.

Roja, punta.

Roldan, arrecife.

Roma, punta.

Romanga, bajo.

Romano, punta, y seno.

Romblon, grupo de islas, isla, pueblo, puerto, y punta.

Roña, islote.

Rosa, punta.

Rosamel, bajo.

Rosario, pueblo, punta, rio, y(del) sierra.

Rosas, punta.

Rubi, bajo.

Rubido, ensenada.

Rumban, punta.

Rungus, punta.

Saac, punta.

Saae, isla.

Saamong, monte.

Sabago, barrio.

Sabalay, banco.

Saban, punta.

Sabang, punta, y rio.

Sablay, punta. 
Sablayan, fondeadero, puehlo, y punta. Saboncogon, punta.

Saboon, islit.

Saboriog, seno.

Saboruco, punta.

Sabtan, islils.

Sacaan, punta.

Sacalagayan, isla, y punti.

Sacaron, sello.

Sacay-sacay, punta.

Saccol, isla.

Saco de Negros, ensemarla.

Sacripante, monte.

Sagal, punta.

Sagao, bantay.

Sagarayan, fondeadero, y islat.

Sagasay, isla.

Sagausaun, punta, y rio.

Sagay, puelslo, y punta.

Sagcadoc, punta.

Sagnay, punta.

Saguing-saguing, punta.

Sagumay, punta.

Saisain, punta.

Sal, islas.

Salacot, punta.

Salado, islas.

Saladongon, arroyo.

Salamague, rio.

Salanan, junta.

Salaquio, rio.

Salasala, punta.

Salauan, punta.

Saley, punta.

Salimbal, punta.

Salimbubuc, isletas.

Salin, rio.

Salinas, torre.

Salincapaoraan, punta.

Salipin, islas.

Salkulakit, isla.

Sallao, punta.

Salog, rio.

Saloganbanug, punta.

Salomague, isla, puerto, y lunta.

Salong, punta.

Saloro, islat.

Saltan, rio.

Saluan, isla.

Salude, islas.

Salug, rio.

Salvador, fondeadero.

Salvaria, isla, y punta.

Samagurabe, arroyo, punta, y seno.
Samal, isla, y villaje.

Samalang, estero.

Samang, punta.

Samar, isli.

Sambabuas, isla.

Sambaton, punta.

Samblaya, punta.

Samboanguita, punta.

Samboan, pueblo.

Sambugtur, rio.

Sambulauan, monte, puerto, y punta.

Samoro, punta.

Samoyan, rio.

Sampinit, laguna.

Sampinitan, estero.

Samquel, rio.

Samulit, seno.

Samur, isla.

Samuro, junta.

Sanaga, rio.

San Agustin, (al)(), ensenada, y pueblo.

San Andres, islas, puerto, y punta.

San Andres, or Maragat, isleta.

San Antonio, ensenarla, islas, monte, y puelilo.

Sanayon, pueblo.

San Bangha, nonte.

San Bernardino, (de) estrecho, y isla.

San Carlos, pueblo.

Sanco, punta.

Sancol, puerto, y punta.

San Cristobal, monte.

San Diego, punta.

San Dionisio, puel,lo.

Sandul, junta.

Sandungan, punta.

San Enrique, pueblo, y rio.

San Esteban, jueblo, y puerto.

San Fabian, pueblo.

San Fernando, bajo, monte, pueblo, puerto, y puntu.

San Fernando, or Panungu, isla.

San Francisco, pueblo.

San Francisco del Monte, puello, y rio.

Sanga, puerto, y punta.

San Gabriel, punta.

Sangasag, pico.

Sanga Sanga, isla, y punta.

Sangay, punta.

Sangbauen, monte.

Sangboy, islas.

Sangilan, punta.

Sangley, punta.

Sangputan, punta. 
Sangubon, punta.

Sanguisiapo, i.sla.

Sanguitan, punta.

Sanig, junta, rio, y seno.

San Ildefonso, calu, y pueblo.

San Isidro, barra, monte, puehlo, y seno.

San Jacinto, pueblo, puerto, y punta.

San Joaquin, pueblo.

San Jose, pueblo, y punta.

San Juan, pueblo, punta, rio, y villaje.

San Juanico, (de) estrecho.

San Julian, ensenada, pueblo, y puerto.

San Martin, purta.

San Mateo, fomlealero, pueblo, y rio.

San Miguel, Jahia, isla, pueblo, y puerto.

San Narciso, pueblo.

San Nicolas, bajos, y pueblo.

San Pablo, monte, y pueblo.

San Pascual, pueblo.

San Pedriño, punta.

San Pedriño, or Pagapas, ensenada.

San Pedro, pueblo, punta, y villaje.

San Pedro y San Pablo, bahia.

San Pedro Tunasan, pueblo.

San Policarpo, villeta.

Sanqui, punta.

San Rafael, pueblo, y punta.

San Remigio, pueblo.

San Ricardo, isla, y pueblo.

San Salvador, isla, y pueblo.

San Sebastian, pueblo, y rio.

Sanson, ensemada.

Santa Ana, pueblo, y puerto.

Santa Bita, villaje.

Santa Catalina, pueblo.

Santa Cruz, ensenada, fontearlero, isla, puerto, punta, y villaje.

Santa Elena, rio.

Santa Filomena, bajo.

Santa Lucia, pueoio.

Santa Margarita, pueblo.

Santa Maria, comandancia, islote, pueblo, y puerto.

Santa Monica, pueblo.

Santana, punta.

Santander, pueblo.

Santa Rita, monte, y pueblo.

Santa Rosa, monte, y pueblo.

Santiago, cabo, isla, monte, pueblo, y juerto.

Santo Domingo, bahia, pueblo, y punta.

Santo Tomas, monte, pueblo, puerto, y punta.

San Vicente, pueblo, y puerti.
Sanz, islt.

Sapa, arroyo, y rio.

Sapao, isla, y pueblo.

Sapenitan, punta.

Sapian, ensenarla, pueblo, y punta.

Sapio, punta.

Sapo, puerto.

Saponga, or Mainit, lago.

Saptang, isla.

Sarangani, bahia, islak, punta, y volcan activo.

Sarap, monte, y junta.

Sarapsap, islaw, y pueblo.

Saravia, pueblo.

Sariaya, pueblo.

Sarmiento, punta.

Sarva, punta.

Sarvo, rio.

Sasa, islote.

Sasaigan, punta.

Sataco, punta.

Satioldkit, islas.

Sayao, ensenarla.

Sauahon, punta.

Sauan, punta, y rio.

Sauang, or Quinamaligan, isla.

Saud, seno.

Sauras, punta.

Schuck, piedra.

Scolt, punta.

Seamer, punta.

Sean, grupo de islas.

Sebungan, punta.

Secam, isla.

Seco, or Pontud, lranco.

Secubun, isla.

Segyam, islas, y punta.

Sekelapit, punta.

Selim, grupo de islas.

Selungan, punta.

Semaruga, punta.

Semba, estero.

Semeneblen, monte.

Sementera, villaje.

Semerara, islas, y pueblo.

Senogan, pueblo.

Sepangow, bahia.

Separacion, punta.

Serantes, bajo.

Sevilla, pueblo.

Shell, isla.

Shirt, punta.

Siacle, punta.

Sialat, punta. 
Siapa, isla.

Siapar, punta.

Siapon, fondearlero.

Siaran, punta.

Siargao, isla, y paso.

Siasan, punta.

Siaton, pueblo, punta, y rio.

Siay, estero.

Siayan, isla.

Sibago, islas.

Sibajay, rio.

Sibala, monte, punta, y rillaje.

Sibale, isla.

Sibali, punta.

Sibaloc, isla.

Sibalon, pueblo, y rio.

Sibanoc, isla.

Sibaon, ensenada.

Sibaton, isletas.

Sibauan, punta.

Sibaul, pueblo.

Sibay, isletas, y pueblo.

Sibayel, rio.

Sibbu, or Siffu, rio.

Sibijan-dacula, isla.

Sibolon, isletas.

Sibonga, pueblo, puerto, y punta.

Sibono, punta.

Sibuay-buay, rio.

Sibucao, punta.

Sibuco, arroyo, ensenada, y punta.

Sibug, villaje.

Sibugay, bajo, isla, y monte.

Sibuguey, isla, monte, y seno.

Sibukin, punta.

Sibulan, isla, puelolo, y punta.

Sibuluac, isla.

Sibuluac-babay, isla.

Sibuluac-lalaqui, isla.

Sibutu, islas.

Sibuyan, islas, monte, y pueblo.

Sibuyan, or Guitinguitin, monte.

Sicaba, pueblu.

Sicayac, punta.

Sicogon, isla, punta, y rio.

Sicot, or Jervois, punta.

Sidang-dang, colina.

Siep-blut, punta.

Siete Pecados, grupo de islas.

Sigaboy, fondeadero, isla, monte, y pueblo.

Sigat, punta.

Sigayan, punta, rio, y seno.

Sigbuye, islas, y paso.
Siglao, punta.

Sihan, punta.

Sila, punta.

Silacay, punta.

Silaga, rio.

Silagui, punta.

Silaguin, ensenada, y punta.

Silancapo, punta.

Silanga, bahia, monte, punta, y villeta.

Silangan, puntil.

Silango, punta.

Silangon, punta.

Silanguin, ensenada, puerto, y punta.

Silaqui, isla.

Silat, islas.

Silauan, bajo.

Silay, monte, y pueblo.

Silik, estero.

Silingan, monte.

Silino, isla.

Silla, islas, monte, y (de la) punta.

Silla del Sabani, monte.

Silo, rio.

Silonay, isla, y rio.

Silongos, islas.

Silsitoan, isla.

Siluban, rio.

Silunga, punta.

Simaga, punta.

Simagup, bahia.

Simahagan, rio.

Simala, punta.

Simaluc, islas.

Simanahan, arrecife, y canal.

Simanale, (de) canal, y fondeadero.

Simanuga, punta.

Simara, islas.

Simauc, estero, y punta.

Similon, isla.

Simisa, islas.

Simo, banco.

Simonog, isla.

Simoyan, rio.

Simuay, rio.

Simulao, rio

Simutu, pueblo.

Sinabu, rio.

Sinagbuan, islas.

Sinangatan, punta, y seno.

Sinapsapan, punta.

Sinaragan, rio.

Sinauat, lago.

Sinay, punta, y villaje.

Sindan, pueblo. 
Sindandan, punta.

Sindangan, pueblo, punta, y s.uo.

Sinebaran, arroyo.

Singaan, islit.

Singuan, laguna.

Sinipay, isla.

Sinipay Diutay, islote.

Sinlian, punta.

Sinobughan, isla.

Sinocalan, rio.

Sinoga, punta.

Sinolo, rio.

Sinonoc, punta, y rio.

Sinonog, islas.

Sinti, punta.

Sintogo, rio.

Sinulon, grupo de islas.

Sioc, puerto, y punta.

Siocon, ensenala, y punta.

Sipac, islas, pueblo, y rio.

Sipaca, punta.

Sipaloy, ensenada.

Sipandin, pueblo.

Sipang, isla.

Siparay, islas.

Sipuaoc, pico.

Sipungut, isla.

Siquijor, isla, y pueblo.

Sir James Brooke, punta.

Siraguay, punta, y rio.

Sirilian, punta.

Sirinao, or Sepulcro, isla.

Siringo, isla,

Siruguay, rio.

Siruma, isla, pueblo, y punta.

Sirun, isla.

Sisiman, ensenada, y punta.

Sisipan, punta.

Sisiran, puerto.

Situma, punta.

Siyt, puerto.

Slanlake, isla.

Slip, punta.

Soan-soan, estero, y punta.

Sobang, rio.

Soca-Vilar, rio.

Sogod, ensenada, y pueblo.

Soguicay, ensenada, y isleta.

Sojoton, islote, y punta.

Sola, rio.

Solana, pueblo.

Solasa, villaje.

Solaton, isla.

Solbec, seno.
Solitario, islas, y monte.

Solot punta.

Solotsolot, or Lapog, bahia, fondeatero, y punta.

Somagui, rio.

Sombocogon, enrenarla.

Sombrero, isla, monte, y punta.

Son-buaya, rio.

Songan Maluja, estero.

Songan Tulac, estero.

Sorpresa, estero.

Sorsogon, provincia, pueblo, y puerto.

Sostoa, bajo.

Sout-Soutan, seno.

Steep, punta.

Sua, monte, punta, y seno.

Suac, punta.

Suague, rio.

Sual, monte, pueblo, y puerto.

Suay, pueblo.

Subaan, ensenarla, punta, y villeta.

Subaang, pueblo, y punta.

Subag, punta.

Subay, villaje.

Subic, pueblo, puerto, y punta.

Subuj, punta.

Subunguin, punta.

Sudeste, monte.

Sudoeste, punta.

Suetan, rio.

Sugac, isla, y punta.

Sugot, ensenarla.

Sugut, rio, seno, sierra, y villa'e.

Sujac, punta.

Sula, (de) paso, puerto, y junta.

Sulade, isla.

Sulanan, rio.

Sulat, ensenada, pueblo, y punta.

Sulauang, punta.

Sulican, islas.

Sultan, monte.

Suluan, islas.

Sulung, punta.

Sumacap, villaje.

Sumag, pueblo.

Sumangul, punta.

Sumay, villaje.

Sumban, punta.

Sumba sumba, isla.

Sumilon, isla.

Sumuhit, punta, y seno.

Suncan, isla.

Sunco, estero.

Sungay, cordillera. 


\section{Sungi, punta.}

Sunlian, punta.

Sunlung, rio.

Suoc, pueblo.

Sur, punta.

Suribao, punta, y rio.

Surigao, (de) estrecho, fondeadero, 1neblo, puerto, y punta.

Susan, isla.

Suso, villeta.

Susun-dalaga, monte.

Suyuc, rio.

Syoin, punta.

Taal, barra, ensenada fonteadero, lacruna, pueblo, y rolcan activo.

Taancan, $o r$ Ninipo, puntil.

Tabac, isla.

Tabaco, fondeadero, puelslo, y seno.

Tabag, ensenada, y junta.

Tabagang, rio.

Tabanel, rio.

Tabango, seno.

Tabantalan, punta.

Tabay, lunta, y rio.

Tabian, punta.

Tabiauan, rio.

Tabicu, junta.

Tabid, estero, y punti.

Tabiggindannan, villaje.

Tabigon, pueblo.

Tabigui, punta, y villaje.

Tabinay, rio.

Tabinay Grande, punta.

Tabin Chico, ensenalia.

Tabin Grande, ensenada.

Tabipanan, punta.

Tabiqui, punta.

Tabirauan, rio.

Tabla, punta.

Tablac, rio.

Tablas, islas, y (de) paso.

Tablazo, or Marlanga, monte.

Table, rin.

Taboc, isla.

Tabogon, puelso.

Tabolotan, ensenala, y punta.

Tabon, isla, y punta.

Tabonan, punta.

Tabones, (de los) isla.

Tabong, villaje.

Tabu, punta.

Tabuan, banco, y rio.

Tabubunga, villeta.
Tabugun, isla.

Tabulian, punta.

Tabulion, monte.

Tabulunga, islas.

Tabun, punta.

Tabunan, punta.

Tabunao, punta.

Tabungao, estero, y rancheria.

Tabuntabun, punta.

Taburan, ensenada, y punta.

Tabuyaca, isla.

Tacbolo, isleta.

Tacbolulu, villaje.

Tacbubuc, islas.

Tacbuc, punta.

Tacligan, junta.

Tacloban, capital, y puertu.

Taclogan, selno.

Tacnipa, punta, y villaje.

Tactacan, prunta, y seno.

Tactay, punta.

Tacubanhan, isla.

Tacud-pabunuan, islas.

Tadloy, punta.

Tadmonan, punta.

Taebun, (1e) canal.

Tagabulin, puerto.

Tagalan, rio.

Tagalinog, isla.

Tagampul, isla.

Taganilao, punta.

Tagao, islas.

Tagapangan, punta.

Tagapula, isla.

Tagauayan, isla.

Tagavivi, ranclueria.

Tagaytay, cordillera.

Tagba, punta.

Tagbabas, isla.

Tagbauan, punta.

Tagbayug, (de) bahia.

Tagbilaran, capital, estero, y (de) jaso.

Tagbin, junta.

Tagdaranas, i.slas.

Tagdugan, ensenarla.

Tagil, islas.

Tagiran, arroyo, punta, y seno.

Tagjaligue, or Jaligue, junta.

Tagmuna, punta.

Tagnipa, estero.

Tagolo, punta.

Tagoloan, pueblo, y rio.

Tagonita, villeta.

Tagpoc, rio. 
Tagtaba, punta.

Tagtada, punta.

Tagtagan, isla.

Taguadian, isla.

Taguan, seno.

Tagubanhan, isla.

Tagudin, yeblo.

Taguictig, seno.

Taguig, bahia, y puehlu.

Taguigoron, arroyo.

Taguinting, rio.

Taguisian, punta.

Taguitic, arroyo.

Tagulaya, punt: .

Taguluan, rin.

Tagum, rio.

Taguranpan, ris.

Tagusan, punta.

Tagutu, isla.

Taguus, punta.

Taguvan, punta.

Tahing-Tahing, isli.

Tahon, punta.

Tahuruc, punta.

Tailon, islas.

Tai-Tay, isleta.

Tajao, Jajo, y punta.

Tajiran, ensenada, y punta.

Tajo, rio, y villaje.

Tajud, punta.

Tajun-tajun, isla.

Takela, isla.

Talabaan, isla, y rio.

Talabajan, punta.

Talaban, isla, y villaje.

Talabasi, junta, y rio.

Talabe, rio.

Talabon, isleta.

Talacanen, isleta.

Talagib, punta.

Talaguilong, puerto.

Talagutun, fondearlero.

Talairan, punta.

Talaji, monte, y punta.

Talajit, or Mesa, isla.

Talamasig, rio.

Talampulan, islas.

Talamtan, banco.

Talaona, villeta.

Talaran. islas.

Talavera, lago, y pueblo.

Talayan, estero, y montes.

Talayo, punta.

Talcauayan, ensenada.
Talibon, puehln.

Talicud, isla.

Talim, isla, y junta.

Talin, ensenarla, fondeatero, monte, $y$ punta.

Talinas, islas, y punta.

Talindac, villeta.

Talinglin, enrenarla, y punta.

Talintig, islote.

Talinting, islote, $y$ pmint.

Talipanan, monte, y punta.

Taliptipan, punta.

Talisain, fondearlero, y ним.

Talisay, monte, jnelslo, pminta, rio, y seno.

Talisaya, punta.

Talisayan, unnte, pueblo, punta, y rio.

Talong, islis.

Talonpisa, islas.

Taluay, rio.

Taluc, islas.

Talucdangan, rio.

Talucluc, islote.

Talulacuban, fiserte.

Tamala, rio.

Tambabo, punta.

Tambadon, senu.

Tambagaan, iskil.

Tambagan, grupo de islas.

Tambalan, punta.

Tambalanan, isleta.

Tambaloangan, canal.

Tambang, puerto, y punta.

Tambanguin, villaje.

Tambaron, isla.

Tambilambi, punta.

Tambilimay, isla.

Tambiluanga, canal, y isla.

Tambisan, punta.

Tambo, villaje.

Tambobo, punta.

Tambog, punta.

Tamboloton, ensenarlax.

Tambon, isla.

Tambongon, islas.

Tamboron, isla.

Tambu, isla.

Tambulan, villeta.

Tambulian, isla, y punta.

Tambunan, punta.

Tambun Sigumbal, puebl..

Tami, punta.

Tamoayac, monte, y peblo.

Tamontaca, fuerte, y pueblo. 
Tam-Tet.

Tampan, estero.

Tampa-tampa, isla.

Tampel, isleta.

Tanabaay, isla.

Tanaban, viliaje.

Tanagon, punta.

Tanaguin, rio.

Tanao, monte, y ris.

Tanauan, pueblo, y rio.

Tanaun, pico.

Tanay, pneblo.

Tancan, islar.

Tancolaluan, isla.

Tancojon, punta.

Tanda, rio, y villaje.

Tandabato, islas.

Tandag, pueblo, y punta.

Tandog, isla.

Tandu, monte, puehlo, y junta.

Tandubas, (de) eanal, y islas.

Tandusahasa, paso.

Tang, punta.

Tangad, isla.

Tangdad Libucan, iwla.

Tangalan, pueblo.

Tangantangan, pun ‘.

Tangat, iwleta.

Tangday, rio.

Tanglao, rio.

Tanglar, punta.

Tangon, punta.

Tanguiguiton, punta.

Tanguingui, islas.

Tangulung, senr.

Tanhan, villaje.

Tanjas, punta.

Tanjong Banga, "abo.

Tanjong Tando-tao, calo.

Tanjong Tavo-Tavo, cabo.

Tanobon, isla.

Tañon, (del) estrecho, junta, y villaje.

Tanquique, piedra.

Tantanang, semo.

Tantauayan, monte.

Tantongen, pico.

Tanuan, punta, y seno.

Taos, seno.

Tapaan, banev, islas, y paso.

Tapac, punta.

Tapao, monte, y punta.

Tapian, monte, y punta.

Tapiantana, islas.

Tapilon, puntal.
Tapitan, punta.

Tapiutan, isla.

Tapul, islas.

Tapulan, punta.

Taquisian, punta.

Tara, islas.

Tarabang, rio.

Tara-cai-a-wan, isla.

Tarangnan, pueblo.

Taranosa, punta.

Tarataha, punta.

Tara Tara, isla, punta, y seno.

Tarataro, islote.

Tarbon, estero.

Tarigtig, punta.

Tarlac, capital, y rio.

Tarol, islas.

Taron, punta.

Taruc, islas.

Tasiran, punta.

Tataan, islas, y puerto.

Tatabuban, seno.

Tataidaga, punta.

Tatalan, isla.

Tatantalang, punta.

Tataran, isla.

Tatus, islote.

Tauasan, monte.

Tausan, rio.

Tavialan, estero.

Taviran, loma.

Tawi-tawi, bahia, y gropro de islas.

Tayabas, punta, rio, y seno.

Tayasan, pueblo, y punta.

Taytay, bahia, islas, pueblo, y junta.

Teca, punta.

Teguit, junta.

Teinga, islas.

Teinga Laguit, isla.

Teipona, isla.

Temple, isla.

Templo, isla.

Tenabian, isleta.

Tendido, monte.

Tenga-lakit, isla.

Tengolan, isla.

Teodoro, punta.

Teolong, isla.

Teomabal, isla.

Terisan, isla.

Termaba, islas.

Ternate, pueblo, y seno.

Tetas de Catainga, nonte. 
Tetas de Santa, monte.

Tetuigpan, esteru.

Tiain, punta.

Tibao, or Calapan, punta.

Tibay, rio.

Tibbyon, ensenada.

Tibi, villaje.

Tibiao, pueblo.

Tibungoy, punta.

Ticala, islas.

Ticao, isla, (de) paso, y seno.

Ticlin, islas, y punta.

Ticlines, (de) estrecho, y grupo ile islas. Tictavun, canal, y isla.

Ticuauan, punta.

Ticul, isla.

Tigalan, rio.

Tigapan, rio.

Tigasao, punta.

Tigbaon, islas.

Tigbauan, monte, pueblo, punta, y rio.

Tigdos, isla.

Tigmanica, rio.

Tigna, rio.

Tigon, rio.

Tigona, villaje.

Tigpuluan, rio.

Tiguegarao, villaje.

Tiguias, punta.

Tiguijan, punta.

Tiguilabun, isla.

Tiguis, punta.

Tiguma, villaje.

Tigungun, or Calupag, isla.

Tiji-Tiji, banco, y islotes.

Tilat, or Buena Vista, pueblo.

Tilig, puerto, punta, y villaje.

Timaco, colina, $\mathrm{y}$ isla.

Timacolo, punta.

Timbungan, islas.

Timonim, rio.

Timoragat, rio.

Timpasan, islas.

Timuto, fondeadero.

Tinaan, fondeadero, punta, y villeta.

Tinabagsan, punta.

Tinaca, punta.

Tinacos, isla.

Tinactadan, punta.

Tinaga, islas.

Tinagbog, rio.

Tinagbud, rio.

Tinagilman, ensenada.
Tinago, riı.

Tinagoan, punta.

Tinagtadan, punta.

Tinagutman, fondeadero, punta, y seno.

Tinajuagan, punta.

Tinalay, rio.

Tinalisayan, isla.

Tinambacan, villeta.

Tinang, islas.

Tinaoyan, punta.

Tincalan, islote.

Tincansan, isla.

Tincasan, punta.

Tingarac, pueblo.

Tingbabang, rio.

Tingib, punta.

Tingloy, ensenada.

Tiniguibin, ensenarla.

Tinipgan, punta.

Tinoc, rio.

Tintiman, ensenada, isla, y punta.

Tinuibo, isla.

Tinutungan, isla.

Tiolas, pueblo, y rio.

Tiquias, punta.

Titunod, rio.

Tobian, punta.

Tocai, monte.

Tochen, rio.

Togal, isla.

Tolan, punta.

Toledo, pueblo.

Tolon, ensenarla, y pueblo.

Tolong, rio.

Tolosa, pueblo.

Tomaan, arrovo.

Tomasa, isla.

Tomonton, punta.

Ton Equet, punta.

Tonga, punta.

Tongo, punta.

Tongon, punta.

Tonguil, punta.

Ton Libit, punta.

Tonquil, isla, y punta.

Ton Sagui, punta.

Ton-Sandungun, canal.

Tonseque, punta.

Toog, seno.

Toquil, grupo de islas.

Toreno, rio.

Torre, islote.

Torreras, villeta.

Totoog, punta.

$$
\text { S. Doc. } 228
$$




\section{Tow-Uai.}

Townsend, punta.

Tozera, pi(').

Traidora, lahia.

Transito, villaje.

Trapichihan, punta.

Treacher, junta.

Tres Picos, isla, y monte.

Tres Reyes, isletos, y monte.

Triple Cima, isla.

Tristan, monte.

Tritagnun, hantay.

Triton, islote.

Trueno, bajo.

Tuad, isla.

Tuagan, rio.

Tuagayan, rio.

Tubabal, isla.

Tubabao, isla.

Tubabat, islote.

Tubajon, isla, y pueblo.

Tubal, fondeadero.

Tubalubac, isla.

Tubay, puelilo, y rio.

Tubaya, rio.

Tubayas, rio.

Tubiajon, pueblo, y punta.

Tubig, pueblo, y punta.

Tubigan, isla.

Tubigon, pueblo, y punta.

Tubile, ensenada, y punta.

Tubingantan, punta.

Tublajon, punta.

Tubotubo, isla.

Tubu, fondearlero.

Tubungan, pneblo.

Tuburan, ensenada, pueblo, punta, y rio.

Tubu-tubu, isla.

Tucalan, isla.

Tucbi, rio.

Tuc Tuc, punta.

Tucuran, fondeadero, y villaje.

Tudela, pueblo.

Tugapangan, punta.

Tugas, punta.

Tugasan, punta.

Tugbugun, punta, y rio.

Tugdao, villeta.

Tugsaban seno.

Tuguban, punta.

Tuguin, punta.

Tuguis, pueblo.

Tugurau, punta.

Tuhinanaun, isla.

Tujud, isleta, y punta.
Tula, estero.

Tulayan, fondeadero, $\mathrm{y}$ islas.

Tulian, islas.

Tuligapo, punta.

Tulipan, monte.

Tulnalutan, isla.

Tulu, islas.

Tulugan, islas.

Tulunanaun, isla.

Tuluran, isla.

Tumaco, punta.

Tumadgo, punta.

Tumaga, rio.

Tumagsa, punta.

Tumaguin, isla.

Tumalaytay, fuerte, isleta, y punta.

Tumalintigan, punta.

Tumalion, villaje.

Tumalung, seno.

Tumalutan, isla.

Tuman, punta.

Tumanao, puerto.

Tumanda, punta.

Tumango, pnerto, or Dilasac, ensenada.

Tumaquin, isla.

Tumaras, punta.

Tumatanguis, monte.

Tumauini, pueblo.

Tumbadon, punta.

Tumbaga, punta.

Tumbao, fuerte, junta, rio, y villaje.

Tuminanayum, islas.

Tumolan, islas.

Tumulac, punta.

Tuna, ensenada, y fondeadero.

Tunao, isla.

Tuncalan, isla.

Tundalara, monte.

Tungalo, villaje.

Tungao, punta.

Tunog, monte.

Tuntumin, rio.

Tuntung, punta.

Tuog, villaje.

Tuquian, punta.

Turung, bahia, y isleta.

Tusang Bongao, canal, y isla.

Tutu, seno.

Tutuban, ensenata.

Tutukipa, isla.

Tuturungan, isleta.

Uag, punta.

Uaidajon, isla. 
Uanivan, isla.

Uban, punta.

Ubay, putblo, y punta.

Ubian, fonteatero, $y$ islas.

Ubunan, rio.

Udlud, punta.

Ugamut, isla.

Ugbun, punta.

Uguis, islote, y punta.

Ugut, rio, y villeta.

Uinas, punta.

Ujut, rio.

Ulan, bantay.

Ulancaya Abdul, monte.

Ulian, rio.

Uling, monte.

Ulip, rio.

Uloc, punta.

Ultra, isla.

Ulugan, or Banog, hahia, y fondeadero.

Ulut, rio.

Uluulu, punta.

Umabay, punta.

Umalantin, punta.

Uman, punta.

Umanian, punta.

Umanun, punta.

Umirey, rio.

Unamao, isla.

Union, provincia, y villaje.

Unip, isla.

Unisan, islotes, y puteblo.

Upala, rio.

Upata, rio.

Urdaneta, monte, y pueblo.

Ursula, isla.

Usada, islas.

Uson, ensenarla, islas, pueblo, punta, y seno.

Usunan, punta.

Utabe, ensenada, rio, y visita.

Uto, punta.

Uwaan, isla.

Uyungan, rio.

Vabanog, punta.

Valencia, pueblo.

Valentin, punta.

Valladolid, pueblo.

Valverde, rio, y villeta.

Varadero, ensenada.

Vasig, rio.
Vay, punta.

Verbena, (de) canal.

Verde, islas, pasos, y punta.

Verde del Norte, islas.

Verde del Sur, islas.

Verdes, grupo de islas.

Vicente, isla.

Victoria, monte, r pueblo.

Vigan, capital.

Vigia, monte, puehlo, y punta.

Vilamil, bajo.

Vilanvilan, isla.

Villaba, pueblo.

Villa Hermosa, villeta.

Vallalon, pueblo, r punta.

Villareal, pueblo, y seno.

Vinalo, isleta.

Viñas, rio.

Vinisitajan, punta, y villeta.

Viraviray, isla.

Viri, isla.

Viriato, villeta.

Vitali, or Bungao, seno

Washington, calos.

Weyler, pueblo.

Yambangan, estero.

Yami, isla.

Yangaba, isla.

Yapusan, punta.

Ygasan, punta.

Ylacaon, isla.

Ylin, (de) canal, fomlearlero, jwa, pmeblo,

y punta.

Ynariguen, rio.

Ynatontan, isla.

Ynatoulan, isla.

Ynitao, punta, y villaje

Yog, punta.

Yucatan, ensenada.

Zamboanga, capital, foncleadero, y pueblo.

Zamboanguita, pueblo, y punta.

Zapato Mayor, grupo le islas.

Zapato Menor, grupo de islas.

Zapatos, islas.

Zapote, rio.

Zaragoza, isla, y pueble.

Zarraga, pueblo, y rio.

Zumarraga, canal, isla, y puehlo. 







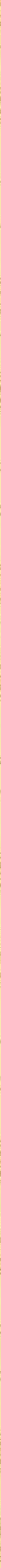




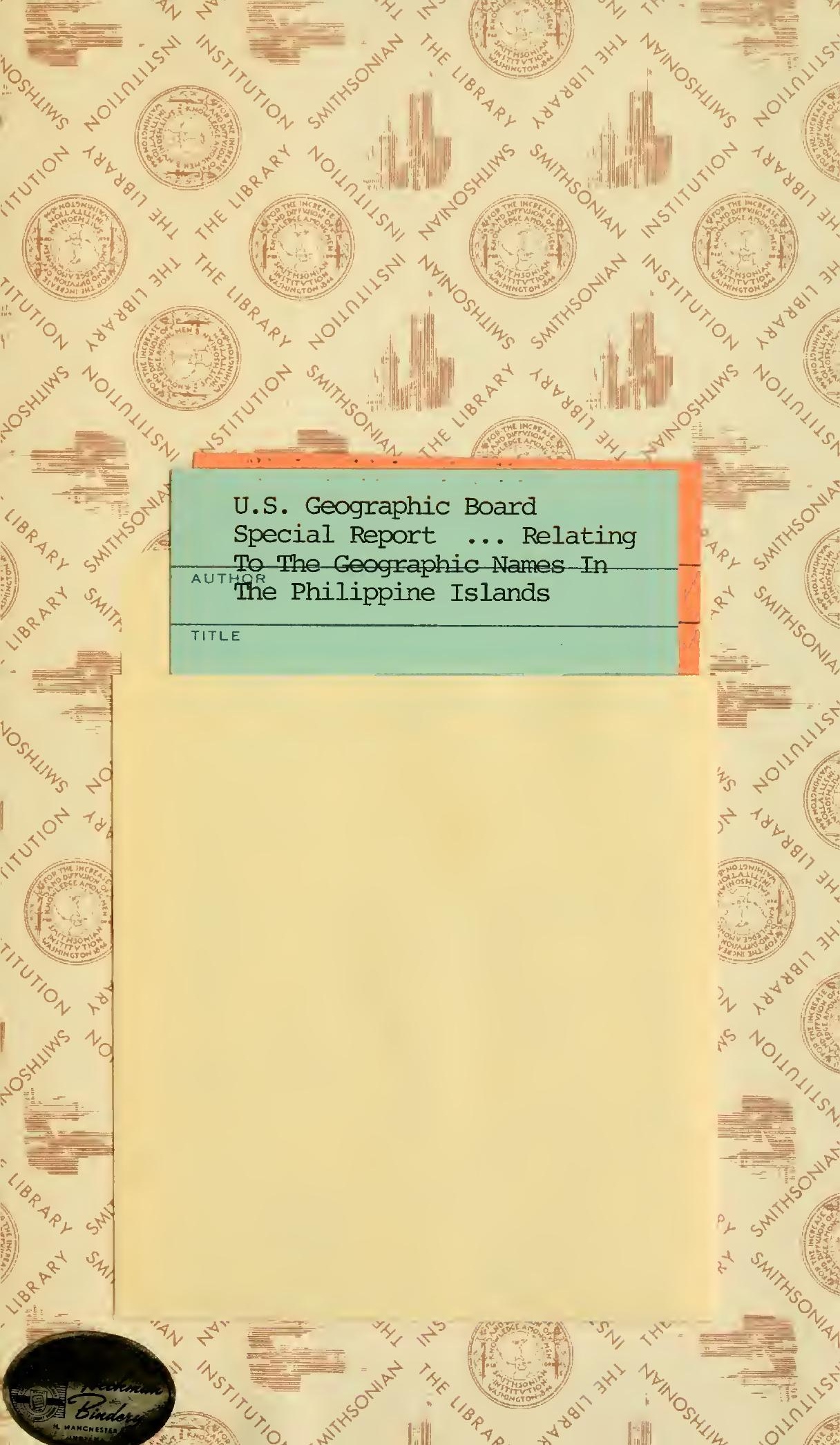




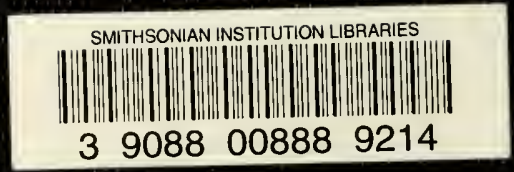

